\title{
Soil organic carbon and land use: Processes and potential in Ontario's long-term agro-ecosystem research sites
}

\author{
Katelyn A. Congreves ${ }^{1}$, Jillian M. Smith ${ }^{2}$, Deanna D. Németh ${ }^{3}$, David C. Hooker ${ }^{4}$, and \\ Laura L. Van Eerd ${ }^{1,5}$ \\ ${ }^{1}$ School of Environmental Sciences, University of Guelph, Ridgetown Campus, Ridgetown, Ontario, Canada NOP \\ 2CO; ${ }^{2}$ Agriculture and Agri-Food Canada, Guelph, Ontario, Canada N1G 4S9; ${ }^{3}$ Ontario Ministry of Agriculture and \\ Food, Ministry of Rural Affairs, Vineland, Ontario, Canada LOR 2EO; and ${ }^{4}$ Department of Plant Agriculture, \\ University of Guelph, Ridgetown Campus, Ridgetown, Ontario, Canada NOP 2 CO. \\ Received 4 October 2013, accepted 12 May 2014. Published on the web 20 May 2014.

\begin{abstract}
Congreves, K. A., Smith, J. M., Németh, D. D., Hooker, D. C. and Van Eerd, L. L. 2014. Soil organic carbon and land use: Processes and potential in Ontario's long-term agro-ecosystem research sites. Can. J. Soil Sci. 94: 317-336. Soil organic carbon (SOC) is crucial for maintaining a productive agro-ecosystem. Long-term research must be synthesized to understand the effects of land management on SOC storage and to develop best practices to prevent soil degradation. Therefore, this review compiled an inventory of long-term Ontario studies and assessed SOC storage under common Ontario land management regimes via a meta-analysis and literature review. In general, greater SOC storage occurred in no-till (NT) vs. tillage systems, in crop rotation vs. continuous corn, and in N fertilizer vs. no N fertilizer systems; however, soil texture and perhaps drainage class may determine the effects of tillage. The effect on SOC storage was variable when deeper soil depth ranges $(0-45 \mathrm{~cm})$ were considered for NT and rotational cropping, which suggests an unpredictable effect of land management on SOC at depths below the plough layer. Therefore, researchers are encouraged to use the presented inventory of nine long-term research sites and 18 active experiments in Ontario to pursue coordinated studies of long-term land management on SOC at depths extending below the plough layer.
\end{abstract}

Key words: Meta-analysis, inventory of long-term experiments, soil organic carbon, no-tillage, crop rotation, nitrogen fertilizer

Congreves, K. A., Smith, J. M., Németh, D. D., Hooker, D. C. et Van Eerd, L. L. 2014. Carbone organique du sol et vocation des terres : mécanismes et possibilités aux sites de recherche de longue haleine sur l'écosystème agricole ontarien. Can. J. Soil Sci. 94: 317-336. Le carbone organique du sol (COS) est indispensable à la productivité de l'écosystème agricole. Il faut concevoir des études de longue haleine pour bien saisir les conséquences de la vocation des terres sur les réserves de COS et élaborer des pratiques exemplaires qui empêcheront la détérioration du sol. Cet article dresse l'inventaire des études à long terme réalisées en Ontario et évalue le stockage du COS en fonction des régimes de vocation des terres courants dans cette province par la méta-analyse et le dépouillement de la documentation. Dans l'ensemble, il y a plus grande conservation du COS avec le non-travail du sol (NT) qu'avec les systèmes incluant des labours, avec les assolements qu'avec la monoculture du maïs, et avec l'usage d'engrais $\mathrm{N}$ que sans fertilisation. Cependant, la texture du sol et éventuellement la classe de drainage pourraient exercer une influence sur les effets du travail du sol. En effet, le stockage du COS varie lorsqu'on tient compte d'une plus grande épaisseur de sol $(0-45 \mathrm{~cm})$ dans l'étude du NT et des assolements, ce qui laisse supposer que l'aménagement des terres a un effet imprévisible sur le COS aux profondeurs supérieures à celle de la semelle de labour. En conséquence, les chercheurs sont incités à utiliser l'inventaire des neuf sites de recherche à long terme et des 18 expériences en cours en Ontario présentés dans cet article pour entreprendre des études coordonnées qui feront ressortir les effets de la gestion des terres à long terme sur la COS, à une profondeur supérieure à celle de la semelle de labour.

Mots clés: Méta-analyse, inventaire des expériences de longue haleine, carbone organique du sol, non-travail du sol, assolement, engrais azotés

A recent environmental commissioner's report identified that $44 \%$ of Ontario's agricultural cropland was at risk of soil erosion and degradation (Ontario 2009). Soil organic carbon (SOC) is crucial for maintaining long-term resilience of agricultural soils and soil productivity. Thus, land management practices that increase or maintain SOC are needed to prevent soil degradation and to maintain crop

${ }^{5}$ Corresponding author (e-mail: lvaneerd@uoguelph.ca). yields and competitiveness of Ontario's agricultural industry. It is therefore essential to understand the effects of land management on SOC in Ontario.

Long-term research provides invaluable insight for quantification of the impact of crop production practices on SOC. The effects of crop production practices on SOC may not be detected with typical timelines of $2-5 \mathrm{yr}$

Abbreviations: CT, conventional tillage system; NT, no-till system; SOC, soil organic carbon 
in short-term projects. For instance, Smith (2004) reported that soil C inputs increased by 20 to $25 \%$, but it took 6-10 yr for a change in SOC to be detected with a $90 \%$ level of confidence. It has been estimated that SOC sequestration rates establish new equilibria in 15-20 yr when comparing no-till (NT) and conventional (CT) practices, and the accumulation of SOC under NT reaches a steady state after 25-30 yr (West and Post 2002; Alvarez 2005). Hence, trials with durations of about $10 \mathrm{yr}$ or more may be most suitable for understanding the dynamics of SOC storage.

International inventories and meta-analyses of longterm soil experiments have been conducted (Powlson et al. 1998; West and Post 2002; Debreczeni et al. 2003). In Canada, a western prairie inventory of long-term soil experiments has been published (Campbell et al. 2012; Lafond and Harker 2012); however, an inventory is lacking for other regions of Canada. Within Ontario, scientists have conducted several long-term field experiments to understand how land management practices such as tillage, crop-rotations, and/or fertilizer affect soil quality parameters such as SOC (Gregorich et al. 1996, 2001; Wanniarachichi et al. 1999; Yang and Kay 2001a, b; VandenBygaart et al. 2002, 2003, 2010; Deen and Kataki 2003; VandenBygaart and Kay 2004; Meyer-Aurich et al. 2006; Angers and Eriksen-Hamel 2008, Yang et al. 2008; Shi et al. 2011; Van Eerd et al. 2014). However, a review of Ontario literature and longterm management practices on SOC is needed to provide relative recommendations on best management practices that maintain or enhance SOC and soil productivity. Therefore, the objectives of this publication were to provide an inventory of long-term research sites in Ontario, assess SOC storage under common Ontario production regimes via a meta-analysis and literature review, and to identify future research needs.

\section{MATERIALS AND METHODS}

Results from published long-term research trials, nonpeer-reviewed reports and by inquiring at universities and governmental research institutions were amalgamated to review the effect of land use management on SOC in Ontario. The research experiments were longterm trials $(>10 \mathrm{yr})$ that were maintained with consistent treatments as well as recently established trials expected to have a long-term duration. Long-term crop cultivar or watershed experiments were excluded.

\section{Meta-analysis}

A dataset was developed from peer-reviewed literature of SOC response to long-term agriculture management in Ontario (Table 1). In order to accurately quantify SOC, the criteria for which literature was used were studies with: $>10 \mathrm{yr}$, SOC on an equivalent basis ( $\mathrm{Mg} \mathrm{C}$ $\mathrm{ha}^{-1}$ ) by depth, and SOC measured to a depth of at least $30 \mathrm{~cm}$. These criteria were similar to those employed by VandenBygaart and Angers (2006). A previous compendium by VandenBygaart et al. (2003) quantified relative change of soil C ( $\%)$ after long-term cropping in Canada, but soil $\mathrm{C}$ data were expressed on an area and soil depth basis ranging from 7.5 to $60 \mathrm{~cm}$ deep. In the present meta-analysis and for each agriculture management practice evaluated, including tillage, crop rotation, and fertilization, the SOC quantities were assembled for three soil depth ranges: the surface layer $(0-10 \mathrm{~cm})$, the plough layer $(0-20 \mathrm{~cm})$, and the deeper profile extending below the plough layer (0-30 to $0-45 \mathrm{~cm})$.

A meta-analysis was conducted to accumulate knowledge across studies. From the dataset, specific treatments at depths $(0-10,0-20,0-45 \mathrm{~cm})$ were paired to determine significant differences in SOC between (a) land use (forest vs. agriculture land); (b) tillage systems (NT or minimal-tillage vs. CT; (c) cropping systems (continuous corn vs. corn in rotation); and (d) $\mathrm{N}$ fertilization $(\mathrm{N}$ application to corn vs. unfertilized control) (Table 1). Each paired data point was presented as SOC on an area basis, and the change in SOC due to the treatment was calculated as a net change $\left(\mathrm{Mg} \mathrm{C} \mathrm{ha}^{-1}\right)$, relative change $(\%)$, and rate of change $\left(\mathrm{g} \mathrm{C} \mathrm{m}^{-2} \mathrm{yr}^{-1}\right)$. The distinct subcategories (i.e., soil depth and management practice) permitted comparisons via meta-analysis (Lipsey and Wilson 2000). The change in SOC based on paired treatments was evaluated using the macro developed by Wilson (2005) for an un-weighted meta-analysis in SAS software (SAS Institute, Inc., version 9.3, Cary, NC). The variations associated with the un-weighted means indicated if the treatment effect on SOC was significant (i.e., if the mean \pm standard error overlapped with zero, then the treatment was not significant). Also within each dataset, a PROC MIXED means comparison (LSD test) with the fixed effect of site was applied to test for site variation and significant differences set at $\alpha \leq 0.05$.

\section{RESULTS AND DISCUSSION}

\section{Inventory of Ontario Long-term Research Sites}

Similar to a Canadian prairie research compilation by Campbell et al. (2012), an inventory of long-term agronomic experiments has been organized and presented herein as a tool for the research community to attain information concerning long-term research sites in Ontario (Table 2). Both published and unpublished research trials have been presented in the inventory (Table 2). The majority of agronomic long-term research in Ontario has focused on field crop rotations involving corn and comparisons of tillage regimes (Table 2), which is representative of the agricultural landscape and management in Ontario. Studies that focused on horticultural production, pasture and livestock grazing systems, organic agriculture and muck soils were not well represented with long-term research in Ontario. As well, some major agricultural production regions in Ontario lack longterm research trials, such as the Niagara region.

To extend Canadian research, data in Table 2 could be merged to the web-based bibliography database Pedosphere.ca - Bibliography of Crop Rotation Studies 
Table 1. A compendium of soil organic C (SOC) data derived from Ontario long-term research sites and organized as paired treatments for (i) land use change, and (ii) crop rotation, (iii) tillage system, and (iv) $\mathbf{N}$ fertilizer strategies

Land use: forest (control) vs. agricultural (treatment) land

\begin{tabular}{|c|c|c|c|c|c|c|c|c|c|}
\hline Site & Control & Treatment $^{\mathbf{z}}$ & $\begin{array}{l}\text { Depth } \\
(\mathrm{cm})\end{array}$ & Study duration (yr) & $\begin{array}{l}\text { SOC control } \\
\left(\mathrm{Mg} \mathrm{ha}^{-1}\right)\end{array}$ & $\begin{array}{l}\text { SOC TRT } \\
\left(\mathrm{Mg} \mathrm{ha}^{-1}\right)\end{array}$ & $\begin{array}{c}\Delta \mathrm{SOC} \\
\left(\mathrm{Mg} \mathrm{ha}^{-1}\right)\end{array}$ & $\Delta \mathrm{SOC}(\%)$ & Reference \\
\hline Ottawa & Forest & corn, till & 30 & $35 \mathrm{yr}-1945-1979$ & 81.5 & 98.5 & 17.1 & 21.0 & $\begin{array}{l}\text { Coote and } \\
\text { Ramsey (1983) }\end{array}$ \\
\hline Ottawa & Forest & corn, till & 30 & $35 \mathrm{yr}-1945-1979$ & 74.9 & 70.8 & -4.1 & -5.5 & $\begin{array}{l}\text { Coote and } \\
\text { Ramsey (1983) }\end{array}$ \\
\hline Ottawa & Forest & grains, till & 30 & 35 yr - 1945-1979 & 244.7 & 207.3 & -37.4 & -15.3 & $\begin{array}{l}\text { Coote and } \\
\text { Ramsey (1983) }\end{array}$ \\
\hline Ottawa & Forest & grains, till & 30 & $35 \mathrm{yr}-1945-1979$ & 64.5 & 56.6 & -8.0 & -12.3 & $\begin{array}{l}\text { Coote and } \\
\text { Ramsey (1983) }\end{array}$ \\
\hline Winchester & Forest & corn, till & 30 & $25 \mathrm{yr}$ - up to 1991 & 122.3 & 96.8 & -25.5 & -20.9 & $\begin{array}{l}\text { Gregorich et al. } \\
\text { (1996) }\end{array}$ \\
\hline Woodslee & Forest & corn, till & 40 & $35 \mathrm{yr}-1959-1994$ & 148.3 & 92.1 & -56.2 & -37.9 & $\begin{array}{l}\text { Gregorich et al. } \\
(2001)\end{array}$ \\
\hline Woodslee & Forest & corn, till, no n & 40 & 35 yr - 1959-1994 & 148.3 & 81.7 & -66.6 & -44.9 & $\begin{array}{l}\text { Gregorich et al. } \\
\text { (2001) }\end{array}$ \\
\hline Woodslee & Forest & c-o-a-a, till & 40 & 35 yr - 1959-1994 & 148.3 & 103.7 & -44.6 & -30.1 & $\begin{array}{l}\text { Gregorich et al. } \\
\text { (2001) }\end{array}$ \\
\hline Woodslee & Forest & c-o-a-a, till, no $\mathrm{N}$ & 40 & $35 \mathrm{yr}-1959-1994$ & 148.3 & 108.8 & -39.5 & -26.6 & $\begin{array}{l}\text { Gregorich et al. } \\
(2001)\end{array}$ \\
\hline Woodslee & Forest & $\mathrm{b}$, till & 40 & $35 \mathrm{yr}-1959-1994$ & 148.3 & 122.8 & -25.5 & -17.2 & $\begin{array}{l}\text { Gregorich et al. } \\
\text { (2001) }\end{array}$ \\
\hline Woodslee & Forest & b, till, no N & 40 & 35 yr - 1959-1994 & 148.3 & 133.2 & -15.1 & -10.2 & $\begin{array}{l}\text { Gregorich et al. } \\
(2001)\end{array}$ \\
\hline Elora & Forest & corn, till & 30 & 29 yr - 1967-1995 & 104.9 & 60.9 & -44.0 & -42.0 & $\begin{array}{l}\text { Wanniarachchi } \\
\text { et al. (1999) }\end{array}$ \\
\hline Elora & Forest & corn, till & 30 & $29 \mathrm{yr}-1967-1995$ & 104.9 & 63.6 & -41.2 & -39.3 & $\begin{array}{l}\text { Wanniarachchi } \\
\text { et al. (1999) }\end{array}$ \\
\hline Bond Head & Forest & cereal, forage, bean & 31 & 68 yr - 1927-1995 & 90.6 & 68.6 & -22.0 & -24.3 & $\begin{array}{l}\text { Ellert and } \\
\text { Gregorich (1996) }\end{array}$ \\
\hline C. Blondeau & Forest & corn, forage & 31 & $25 \mathrm{yr}-1970-1995$ & 136.7 & 109.0 & -27.7 & -20.3 & $\begin{array}{l}\text { Ellert and } \\
\text { Gregorich (1996) }\end{array}$ \\
\hline Delhi & Forest & oat, tobacco & 31 & $\mathrm{~N} / \mathrm{A}^{\mathrm{y}}$ & 55.0 & 24.6 & -30.4 & -55.3 & $\begin{array}{l}\text { Ellert and } \\
\text { Gregorich (1996) }\end{array}$ \\
\hline Edwards & Forest & corn, cereal, forage & 31 & $\mathrm{~N} / \mathrm{A}$ & 133.6 & 42.5 & -91.1 & -68.2 & $\begin{array}{l}\text { Ellert and } \\
\text { Gregorich (1996) }\end{array}$ \\
\hline Exeter & Forest & $\begin{array}{l}\text { corn, cereal, } \\
\text { soybean }\end{array}$ & 31 & $170 \mathrm{yr}-1825-1995$ & 95.3 & 59.2 & -36.1 & -37.9 & $\begin{array}{l}\text { Ellert and } \\
\text { Gregorich (1996) }\end{array}$ \\
\hline Fonthill & Forest & forage, cereal, corn & 31 & $195 \mathrm{yr}-1800-1995$ & 79.6 & 60.9 & -18.7 & -23.5 & $\begin{array}{l}\text { Ellert and } \\
\text { Gregorich (1996) }\end{array}$ \\
\hline Highgate & Forest & corn, soybean & 31 & $\mathrm{~N} / \mathrm{A}$ & 196.6 & 181.5 & -15.1 & -7.7 & $\begin{array}{l}\text { Ellert and } \\
\text { Gregorich (1996) }\end{array}$ \\
\hline Kapuskasing & Forest & forage, cereal & 31 & 53 yr - 1942-1995 & 79.4 & 76.1 & -3.3 & -4.2 & $\begin{array}{l}\text { Ellert and } \\
\text { Gregorich (1996) }\end{array}$ \\
\hline Kemptville & Forest & corn, soybean & 31 & $\mathrm{~N} / \mathrm{A}$ & 81.6 & 56.7 & -24.9 & -30.5 & $\begin{array}{l}\text { Ellert and } \\
\text { Gregorich (1996) }\end{array}$ \\
\hline Panmure & Forest & forage, cereal, corn & 31 & $145 \mathrm{yr}-1850-1995$ & 84.7 & 64.7 & -20.0 & -23.6 & $\begin{array}{l}\text { Ellert and } \\
\text { Gregorich (1996) }\end{array}$ \\
\hline
\end{tabular}


Land use: forest (control) vs. agricultural (treatment) land

\begin{tabular}{|c|c|c|c|c|c|c|c|c|c|}
\hline Site & Control & Treatment $^{\mathbf{z}}$ & $\begin{array}{l}\text { Depth } \\
(\mathrm{cm})\end{array}$ & Study duration (yr) & $\begin{array}{l}\text { SOC control } \\
\left(\mathrm{Mg} \mathrm{ha}^{-1}\right)\end{array}$ & $\begin{array}{l}\text { SOC TRT } \\
\left(\mathrm{Mg} \mathrm{ha}^{-1}\right)\end{array}$ & $\begin{array}{c}\Delta \mathrm{SOC} \\
\left(\mathrm{Mg} \mathrm{ha}^{-1}\right)\end{array}$ & $\Delta \mathrm{SOC}(\%)$ & Reference \\
\hline Plainfield & Forest & forage, cereal, corn & 31 & $91 \mathrm{yr}-1904-1995$ & 105.3 & 62.9 & -42.4 & -40.3 & $\begin{array}{l}\text { Ellert and } \\
\text { Gregorich (1996) }\end{array}$ \\
\hline Ste. Anne & Forest & forage, corn, cereal & 31 & $85 \mathrm{yr}-1910-1995$ & 108.1 & 71.1 & -37.0 & -34.2 & $\begin{array}{l}\text { Ellert and } \\
\text { Gregorich (1996) }\end{array}$ \\
\hline Vineland & Forest & $\begin{array}{l}\text { peach, annual } \\
\text { ryegrass }\end{array}$ & 31 & $145 \mathrm{yr}-1850-1995$ & 56.5 & 40.1 & -16.4 & -29.0 & $\begin{array}{l}\text { Ellert and } \\
\text { Gregorich (1996) }\end{array}$ \\
\hline Winchester & Forest & $\begin{array}{l}\text { corn, forage, } \\
\text { soybean }\end{array}$ & 31 & $105 \mathrm{yr}-1859-1995$ & 127.3 & 65.3 & -62.0 & -48.7 & $\begin{array}{l}\text { Ellert and } \\
\text { Gregorich (1996) }\end{array}$ \\
\hline \multirow[t]{2}{*}{ Woodslee } & Forest & corn, forage & 31 & $\mathrm{~N} / \mathrm{A}$ & 144.0 & 76.1 & -67.9 & -47.2 & $\begin{array}{l}\text { Ellert and } \\
\text { Gregorich (1996) }\end{array}$ \\
\hline & \multicolumn{9}{|c|}{ Crop rotation: corn (control) vs. legume-based rotation (treatment) } \\
\hline
\end{tabular}

\begin{tabular}{|c|c|c|c|c|c|c|c|c|c|c|}
\hline Site & Control & Treatment & $\begin{array}{l}\text { Depth } \\
(\mathrm{cm})\end{array}$ & Study duration (yr) & $\begin{array}{c}\text { SOC } \\
\text { Control } \\
\left(\mathrm{Mg} \mathrm{ha}^{-1}\right)\end{array}$ & $\begin{array}{l}\text { SOC TRT } \\
\left(\mathrm{Mg} \mathrm{ha}^{-1}\right)\end{array}$ & $\begin{array}{c}\Delta \mathrm{SOC} \\
\left(\mathrm{Mg} \mathrm{ha}^{-1}\right)\end{array}$ & $\Delta \mathrm{SOC}(\%)$ & $\begin{array}{c}\Delta \mathrm{SOC} \\
\left(\mathrm{g} \mathrm{C} \mathrm{m}^{-2} \mathrm{yr}^{-1}\right)\end{array}$ & Reference \\
\hline Elora & $\mathrm{cc}$ & $a-a-c-c$ & 10 & $20 \mathrm{yr}-1980-1999$ & 30.0 & 30.1 & 0.1 & 0.2 & 0.3 & Yang and Kay (2001a) \\
\hline Elora & $\mathrm{cc}$ & S-s-c-c & 10 & $20 \mathrm{yr}-1980-1999$ & 30.0 & 29.4 & -0.6 & -1.9 & -2.8 & Yang and Kay (2001a) \\
\hline Elora & $\mathrm{cc}$ & b-rc-c-c & 10 & $20 \mathrm{yr}-1980-1999$ & 30.0 & 31.4 & 1.4 & 4.7 & 7.0 & Yang and Kay (2001a) \\
\hline Elora & $\mathrm{cc}$ & $\mathrm{s}-\mathrm{w}+\mathrm{rc}-\mathrm{c}-\mathrm{c}$ & 10 & $20 \mathrm{yr}-1980-1999$ & 30.0 & 29.7 & -0.3 & -0.8 & -1.3 & Yang and Kay (2001a) \\
\hline Elora & $\mathrm{cc}$ & s-w-c-c & 10 & $20 \mathrm{yr}-1980-1999$ & 30.0 & 32.4 & 2.4 & 8.0 & 12.0 & Yang and Kay (2001a) \\
\hline Ridgetown & cc (no till, no N) & c-s (no till, no N) & 10 & $11 \mathrm{yr}-1995-2006$ & 34.8 & 29.5 & -5.3 & -15.2 & -35.3 & unpublished \\
\hline Ridgetown & cc (no till, no N) & c-s-w (no till, no N) & 10 & $11 \mathrm{yr}-1995-2006$ & 34.8 & 32.5 & -2.3 & -6.6 & -15.3 & unpublished \\
\hline Ridgetown & cc (till, no N) & c-s (till, no N) & 10 & $11 \mathrm{yr}-1995-2006$ & 23.4 & 25.8 & 2.4 & 10.3 & 16.0 & unpublished \\
\hline Ridgetown & cc (till, no N) & c-s-w (till, no N) & 10 & $11 \mathrm{yr}-1995-2006$ & 23.4 & 27.4 & 4.0 & 17.1 & 26.7 & unpublished \\
\hline Ridgetown & cc (no till) & c-s (no till) & 10 & $11 \mathrm{yr}-1995-2006$ & 30.8 & 30.6 & -0.2 & -0.6 & -1.3 & Van Eerd et al. (2014) \\
\hline Ridgetown & cc (no till) & c-s-w (no till) & 10 & $11 \mathrm{yr}-1995-2006$ & 30.8 & 35.4 & 4.6 & 14.9 & 30.7 & Van Eerd et al. (2014) \\
\hline Ridgetown & $\mathrm{cc}(\mathrm{till})$ & c-s (till) & 10 & $11 \mathrm{yr}-1995-2006$ & 24.7 & 24.6 & -0.1 & -0.4 & -0.7 & Van Eerd et al. (2014) \\
\hline Ridgetown & cc (till) & $\mathrm{c}-\mathrm{s}-\mathrm{w}(\mathrm{t}$ till) & 10 & $11 \mathrm{yr}-1995-2006$ & 24.7 & 27.6 & 2.9 & 11.7 & 19.3 & Van Eerd et al. (2014) \\
\hline Woodslee & cc (till) & c-o-a-a (till) & 10 & 35 yr - 1959-1994 & 26.9 & 27.1 & 0.2 & 0.7 & 0.6 & Gregorich et al. (2001) \\
\hline Woodslee & cc (till, no N) & c-o-a-a (till, no N) & 10 & 35 yr - 1959-1994 & 23.1 & 27.8 & 4.7 & 20.3 & 13.4 & Gregorich et al. (2001) \\
\hline Elora & $\mathrm{cc}$ & $a-a-c-c$ & 20 & $20 \mathrm{yr}-1980-1999$ & 58.7 & 58.2 & -0.6 & -1.0 & -3.0 & Yang and Kay (2001a) \\
\hline Elora & $\mathrm{cc}$ & s-s-c-c & 20 & $20 \mathrm{yr}-1980-1999$ & 58.7 & 59.4 & 0.6 & 1.1 & 3.1 & Yang and Kay (2001a) \\
\hline Elora & $\mathrm{cc}$ & b-rc-b-rc-c-c & 20 & $20 \mathrm{yr}-1980-1999$ & 58.7 & 61.3 & 2.6 & 4.4 & 12.9 & Yang and Kay (2001a) \\
\hline Elora & $\mathrm{cc}$ & $\mathrm{s}-\mathrm{w}+\mathrm{rc}-\mathrm{c}-\mathrm{c}$ & 20 & $20 \mathrm{yr}-1980-1999$ & 58.7 & 58.3 & -0.4 & -0.7 & -2.2 & Yang and Kay (2001a) \\
\hline Elora & $\mathrm{cc}$ & s-w-c-c & 20 & $20 \mathrm{yr}-1980-1999$ & 58.7 & 63.5 & 4.7 & 8.1 & 23.7 & Yang and Kay (2001a) \\
\hline Ridgetown & cc (no till, no N) & c-s (no till, no N) & 20 & $11 \mathrm{yr}-1995-2006$ & 63.8 & 55.1 & -8.7 & -13.6 & -58.0 & unpublished \\
\hline Ridgetown & cc (no till, no N) & c-s-w (no till, no N) & 20 & $11 \mathrm{yr}-1995-2006$ & 63.8 & 59.3 & -4.5 & -7.1 & -30.0 & unpublished \\
\hline Ridgetown & cc (till, no N) & c-s (till, no N) & 20 & $11 \mathrm{yr}-1995-2006$ & 46.4 & 52.7 & 6.3 & 13.6 & 42.0 & unpublished \\
\hline Ridgetown & cc (till, no N) & $\mathrm{c}-\mathrm{s}-\mathrm{w}$ (till, no N) & 20 & $11 \mathrm{yr}-1995-2006$ & 46.4 & 54.7 & 8.3 & 17.9 & 55.3 & unpublished \\
\hline Ridgetown & $\mathrm{cc}$ (no till) & c-s (no till) & 20 & $11 \mathrm{yr}-1995-2006$ & 60.2 & 56.9 & -3.3 & -5.5 & -22.0 & Van Eerd et al. (2014) \\
\hline Ridgetown & cc (no till) & $\mathrm{c}-\mathrm{s}-\mathrm{w}$ (no till) & 20 & $11 \mathrm{yr}-1995-2006$ & 60.2 & 66 & 5.8 & 9.6 & 38.7 & Van Eerd et al. (2014) \\
\hline Ridgetown & $\mathrm{cc}(\mathrm{till})$ & c-s (till) & 20 & $11 \mathrm{yr}-1995-2006$ & 48.8 & 49.9 & 1.1 & 2.3 & 7.3 & Van Eerd et al. (2014) \\
\hline Ridgetown & cc (till) & $\mathrm{c}-\mathrm{s}-\mathrm{w}(\mathrm{till})$ & 20 & $11 \mathrm{yr}-1995-2006$ & 48.8 & 53.7 & 4.9 & 10.0 & 32.7 & Van Eerd et al. (2014) \\
\hline Woodslee & cc (till) & c-o-a-a (till) & 20 & $35 \mathrm{yr}-1959-1994$ & 55.6 & 55.9 & 0.3 & 0.5 & 0.9 & Gregorich et al. (2001) \\
\hline Woodslee & cc (till) & c-o-a-a (till, no N) & 20 & $35 \mathrm{yr}-1959-1994$ & 51.4 & 56.9 & 5.5 & 10.7 & 15.7 & Gregorich et al. (2001) \\
\hline Elora & $\mathrm{cc}$, no $\mathrm{N}$ & b-rc-b-rc-c-c & 34 & $20 \mathrm{yr}-1980-2000$ & 77.7 & 81.6 & 3.9 & 5.0 & 19.5 & $\begin{array}{l}\text { Meyer-Aurich et al. } \\
(2006)\end{array}$ \\
\hline
\end{tabular}


Crop rotation: corn (control) vs. legume-based rotation (treatment)

\begin{tabular}{|c|c|c|c|c|c|c|c|c|c|c|}
\hline Site & Control & Treatment & $\begin{array}{l}\text { Depth } \\
(\mathrm{cm})\end{array}$ & Study duration (yr) & $\begin{array}{c}\text { SOC } \\
\text { Control } \\
\left(\mathrm{Mg} \mathrm{ha}^{-1}\right)\end{array}$ & $\begin{array}{l}\text { SOC TRT } \\
\left(\mathrm{Mg} \mathrm{ha}^{-1}\right)\end{array}$ & $\begin{array}{c}\Delta \mathrm{SOC} \\
\left(\mathrm{Mg} \mathrm{ha}^{-1}\right)\end{array}$ & $\Delta \mathrm{SOC}(\%)$ & $\begin{array}{c}\Delta \mathrm{SOC} \\
\left(\mathrm{g} \mathrm{C} \mathrm{m}^{-2} \mathrm{yr}^{-1}\right)\end{array}$ & Reference \\
\hline Elora & $\mathrm{cc}$ & s-s-c-c & 34 & $20 \mathrm{yr}-1980-2000$ & 77.7 & 76.3 & -1.4 & -1.8 & -7.0 & $\begin{array}{l}\text { Meyer-Aurich et al. } \\
\text { (2006) }\end{array}$ \\
\hline Elora & $\mathrm{cc}$ & $s-w-c-c$ & 34 & $20 \mathrm{yr}-1980-2000$ & 77.7 & 80.4 & 2.7 & 3.5 & 13.5 & $\begin{array}{l}\text { Meyer-Aurich et al. } \\
(2006)\end{array}$ \\
\hline Elora & $\mathrm{cc}$ & $\mathrm{s}-\mathrm{w}+\mathrm{rc}-\mathrm{c}-\mathrm{c}$ & 34 & $20 \mathrm{yr}-1980-2000$ & 77.7 & 81.1 & 3.4 & 4.4 & 17.0 & $\begin{array}{l}\text { Meyer-Aurich et al. } \\
(2006)\end{array}$ \\
\hline Elora & $\mathrm{cc}$ & $a-a-c-c$ & 34 & $20 \mathrm{yr}-1980-2000$ & 77.7 & 83.5 & 5.8 & 7.5 & 29.0 & $\begin{array}{l}\text { Meyer-Aurich et al. } \\
\text { (2006) }\end{array}$ \\
\hline Elora & $\mathrm{cc}$ & $a-a-c-c$ & 40 & $20 \mathrm{yr}-1980-1999$ & 81.2 & 78.7 & -2.5 & -3.1 & -12.4 & Yang and Kay (2001a) \\
\hline Elora & $\mathrm{cc}$ & s-s-c-c & 40 & $20 \mathrm{yr}-1980-1999$ & 81.2 & 82.0 & 0.8 & 0.9 & 3.8 & Yang and Kay (2001a) \\
\hline Elora & $\mathrm{cc}$ & b-rc-b-rc-c-c & 40 & 20 yr $-1980-1999$ & 81.2 & 85.6 & 4.3 & 5.4 & 21.8 & Yang and Kay (2001a) \\
\hline Elora & $\mathrm{cc}$ & $\mathrm{s}-\mathrm{w}+\mathrm{rc}-\mathrm{c}-\mathrm{c}$ & 40 & $20 \mathrm{yr}-1980-1999$ & 81.2 & 83.6 & 2.4 & 3.0 & 12.0 & Yang and Kay (2001a) \\
\hline Elora & $\mathrm{cc}$ & s-w-c-c & 40 & $20 \mathrm{yr}-1980-1999$ & 81.2 & 84.6 & 3.4 & 4.2 & 17.1 & Yang and Kay (2001a) \\
\hline Ridgetown & cc (no till, no N) & c-s (no till, no N) & 40 & $11 \mathrm{yr}-1995-2006$ & 107.8 & 84.7 & -23.1 & -21.4 & -154.0 & unpublished \\
\hline Ridgetown & cc (no till, no N) & c-s-w (no till, no N) & 40 & $11 \mathrm{yr}-1995-2006$ & 107.8 & 100.5 & -7.3 & -6.8 & -48.7 & unpublished \\
\hline Ridgetown & cc (till, no N) & c-s (till, no N) & 40 & $11 \mathrm{yr}-1995-2006$ & 73.8 & 81.5 & 7.7 & 10.4 & 51.3 & unpublished \\
\hline Ridgetown & cc (till, no N) & c-s-w (till, no N) & 40 & $11 \mathrm{yr}-1995-2006$ & 73.8 & 89.3 & 15.5 & 21.0 & 103.3 & unpublished \\
\hline Ridgetown & cc (no till) & c-s (no till) & 40 & $11 \mathrm{yr}-1995-2006$ & 104.2 & 96.8 & -7.4 & -7.1 & -49.3 & Van Eerd et al. (2014) \\
\hline Ridgetown & cc (no till) & c-s-w (no till) & 40 & $11 \mathrm{yr}-1995-2006$ & 104.2 & 100.6 & -3.6 & -3.5 & -24.0 & Van Eerd et al. (2014) \\
\hline Ridgetown & cc (till) & c-s (till) & 40 & $11 \mathrm{yr}-1995-2006$ & 79.3 & 80.9 & 1.6 & 2.0 & 10.7 & Van Eerd et al. (2014) \\
\hline Ridgetown & cc (till) & c-s-w (till) & 40 & $11 \mathrm{yr}-1995-2006$ & 79.3 & 87.7 & 8.4 & 10.6 & 56.0 & Van Eerd et al. (2014) \\
\hline Woodslee & cc (till) & c-o-a-a (till) & 40 & $35 \mathrm{yr}-1959-1994$ & 92.1 & 103.7 & 11.6 & 12.6 & 33.1 & Gregorich et al. (2001) \\
\hline Woodslee & cc (till, no till) & c-o-a-a (till, no N) & 40 & 35 yr - 1959-1994 & 81.7 & 108.8 & 27.1 & 33.2 & 77.4 & Gregorich et al. (2001) \\
\hline Ridgetown & cc (no till, no N) & c-s (no till, no N) & 120 & $11 \mathrm{yr}-1995-2006$ & 231.3 & 196 & -35.3 & -15.3 & -235.3 & unpublished \\
\hline Ridgetown & cc (no till, no N) & $\mathrm{c}-\mathrm{s}-\mathrm{w}$ (no till, no N) & 120 & $11 \mathrm{yr}-1995-2006$ & 231.3 & 212.9 & -18.4 & -8.0 & -122.7 & unpublished \\
\hline Ridgetown & cc (till, no N) & c-s (till, no N) & 120 & $11 \mathrm{yr}-1995-2006$ & 161.7 & 169.7 & 8.0 & 4.9 & 53.3 & unpublished \\
\hline Ridgetown & cc (till, no N) & $\mathrm{c}-\mathrm{s}-\mathrm{w}($ till, no N) & 120 & $11 \mathrm{yr}-1995-2006$ & 161.7 & 186.3 & 24.6 & 15.2 & 164.0 & unpublished \\
\hline Ridgetown & cc (no till) & c-s (no till) & 120 & $11 \mathrm{yr}-1995-2006$ & 221.2 & 198.3 & -22.9 & -10.4 & -152.7 & Van Eerd et al. (2014) \\
\hline Ridgetown & $\mathrm{cc}$ (no till) & c-s-w (no till) & 120 & $11 \mathrm{yr}-1995-2006$ & 221.2 & 213.4 & -7.8 & -3.5 & -52.0 & Van Eerd et al. (2014) \\
\hline Ridgetown & $\mathrm{cc}(\mathrm{till})$ & c-s (till) & 120 & $11 \mathrm{yr}-1995-2006$ & 179 & 243.7 & 64.7 & 36.1 & 431.3 & Van Eerd et al. (2014) \\
\hline Ridgetown & cc (till) & c-s-w (till) & 120 & $11 \mathrm{yr}-1995-2006$ & 179 & 170.3 & -8.7 & -4.9 & -58.0 & Van Eerd et al. (2014) \\
\hline \multicolumn{11}{|c|}{ Tillage systems: no till (control) vs. tillage (treatment) } \\
\hline Site & Control & Treatment & $\begin{array}{l}\text { Depth } \\
(\mathrm{cm})\end{array}$ & Study duration (yr) & $\begin{array}{c}\text { SOC } \\
\text { Control } \\
\left(\mathrm{Mg} \mathrm{ha}^{-1}\right)\end{array}$ & $\begin{array}{l}\text { SOC TRT } \\
\left(\mathrm{Mg} \mathrm{ha}^{-1}\right)\end{array}$ & $\begin{array}{c}\left.\Delta \mathrm{SOC}^{-1}\right) \\
\left(\mathrm{Mg} \mathrm{ha}^{-1}\right)\end{array}$ & $\Delta \mathrm{SOC}(\%)$ & $\begin{array}{c}\Delta \mathrm{SOC} \\
\left(\mathrm{g} \mathrm{C} \mathrm{m}^{-2} \mathrm{yr}^{-1}\right)\end{array}$ & Reference \\
\hline Clinton & no till (c-w) & mb till (c-w) & 10 & $22 \mathrm{yr}-1979-2001$ & 40.1 & 36.3 & -3.8 & -9.5 & -17.3 & $\begin{array}{l}\text { VandenBygaart and } \\
\text { Kay (2004) }\end{array}$ \\
\hline Clinton & no till (c-w) & mb till (c-w) & 10 & $22 \mathrm{yr}-1979-2001$ & 14.6 & 14.2 & -0.4 & -2.7 & -1.8 & $\begin{array}{l}\text { VandenBygaart and } \\
\text { Kay (2004) }\end{array}$ \\
\hline Clinton & no till (c-w) & mb till (c-w) & 10 & $22 \mathrm{yr}-1979-2001$ & 29.5 & 27.3 & -2.2 & -7.5 & -10.0 & $\begin{array}{l}\text { VandenBygaart and } \\
\text { Kay (2004) }\end{array}$ \\
\hline Clinton & no till (c-w) & mb till (c-w) & 10 & $22 \mathrm{yr}-1979-2001$ & 32.6 & 30.5 & -2.1 & -6.4 & -9.5 & $\begin{array}{l}\text { VandenBygaart and } \\
\text { Kay (2004) }\end{array}$ \\
\hline Clinton & no till (c-w) & mb till (c-w) & 10 & 19 yr - 1981-1999 & 76.2 & 49.6 & -26.6 & -34.8 & -139.7 & Yang and Kay (2001b) \\
\hline Clinton & no till (c-w) & mb till (c-w) & 10 & $19 \mathrm{yr}-1981-1999$ & 47.7 & 42.7 & -5.0 & -10.5 & -26.4 & Yang and Kay (2001b) \\
\hline
\end{tabular}


Tillage systems: no till (control) vs. tillage (treatment)

\begin{tabular}{|c|c|c|c|c|c|c|c|c|c|c|}
\hline Site & Control & Treatment & $\begin{array}{l}\text { Depth } \\
(\mathrm{cm})\end{array}$ & Study duration (yr) & $\begin{array}{c}\text { SOC } \\
\text { Control } \\
\left(\mathrm{Mg} \mathrm{ha}^{-1}\right)\end{array}$ & $\begin{array}{l}\text { SOC TRT } \\
\left(\mathrm{Mg} \mathrm{ha}^{-1}\right)\end{array}$ & $\begin{array}{c}\Delta \mathrm{SOC} \\
\left(\mathrm{Mg} \mathrm{ha}^{-1}\right)\end{array}$ & $\Delta \mathrm{SOC}(\%)$ & 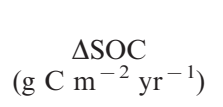 & Reference \\
\hline Clinton & no till (c-w) & mb till (c-w) & 10 & 19 yr - 1981-1999 & 28.7 & 21.5 & -7.2 & -25.1 & -37.8 & Yang and Kay (2001b) \\
\hline Elora & no till (c-s) & $\mathrm{mb}$ till $(\mathrm{c}-\mathrm{s})$ & 10 & $25 \mathrm{yr}-1976-2001$ & 22.9 & 23.6 & 0.7 & 2.9 & 2.7 & Deen and Kataki (2003) \\
\hline Elora & no till (c-s) & mb till (c-s) & 10 & $25 \mathrm{yr}-1976-2001$ & 22.9 & 18.6 & -4.3 & -18.8 & -17.3 & Deen and Kataki (2003) \\
\hline Elora & no till (c-s) & mb till (c-s) & 10 & $25 \mathrm{yr}-1976-2001$ & 22.9 & 23.3 & 0.4 & 1.8 & 1.7 & Deen and Kataki (2003) \\
\hline Elora & no till (c-s) & ch till (c-s) & 10 & $25 \mathrm{yr}-1976-2001$ & 22.9 & 24.4 & 1.5 & 6.5 & 5.9 & Deen and Kataki (2003) \\
\hline Elora & min till $(\mathrm{cc})$ & till $(\mathrm{cc})$ & 10 & $16 \mathrm{yr}-1980-1995$ & 27.3 & 24.7 & -2.6 & -9.4 & -16.0 & $\begin{array}{l}\text { Wanniarachchi et al. } \\
\text { (1999) }\end{array}$ \\
\hline Elora & no till (cc) & $\mathrm{mb}$ till $(\mathrm{cc})$ & 10 & $23 \mathrm{yr}^{\mathrm{x}}$ & 30.5 & 27.5 & -3.0 & -9.8 & -13.0 & Yang et al. (2008) \\
\hline Ridgetown & no till (c-s) & till (c-s) & 10 & $15 \mathrm{yr}-1991-2006$ & 29.2 & 24.5 & -4.7 & -16.1 & -31.3 & Van Eerd et al. (2014) \\
\hline Ridgetown & no till (cc, no N) & till $(\mathrm{cc}$, no $\mathrm{N})$ & 10 & $11 \mathrm{yr}-1995-2006$ & 34.8 & 23.4 & -11.4 & -32.8 & -76.0 & unpublished \\
\hline Ridgetown & no till (c-s, no N) & till $(\mathrm{c}-\mathrm{s}, \mathrm{no} \mathrm{N})$ & 10 & $11 \mathrm{yr}-1995-2006$ & 29.5 & 25.8 & -3.7 & -12.5 & -24.7 & unpublished \\
\hline Ridgetown & $\begin{array}{l}\text { no till (c-s-w, } \\
\text { no } \mathrm{N})\end{array}$ & till (c-s-w, no N) & 10 & $11 \mathrm{yr}-1995-2006$ & 32.5 & 27.4 & -5.1 & -15.7 & -34.0 & unpublished \\
\hline Ridgetown & no till $(\mathrm{cc})$ & till (cc) & 10 & $11 \mathrm{yr}-1995-2006$ & 30.8 & 24.7 & -6.1 & -19.8 & -40.7 & Van Eerd et al. (2014) \\
\hline Ridgetown & no till (c-s) & till (c-s) & 10 & $11 \mathrm{yr}-1995-2006$ & 30.6 & 24.6 & -6.0 & -19.6 & -40.0 & Van Eerd et al. (2014) \\
\hline Ridgetown & no till (c-s-w) & till (c-s-w) & 10 & $11 \mathrm{yr}-1995-2006$ & 35.4 & 27.6 & -7.8 & -22.0 & -52.0 & Van Eerd et al. (2014) \\
\hline Ridgetown & no till (c-s) & ch till (c-s) & 10 & $15 \mathrm{yr}-1991-2006$ & 29.2 & 26.7 & -2.5 & -8.6 & -16.7 & Van Eerd et al. (2014) \\
\hline Woodslee & no till (w-c-s) & mb till (w-c-s) & 10 & $16 \mathrm{yr}-1993-2009$ & 29.4 & 25.6 & -3.8 & -12.9 & -23.8 & Shi et al. (2011) \\
\hline Woodslee & no till $(\mathrm{c}-\mathrm{s})$ & $\mathrm{mb}$ till $(\mathrm{c}-\mathrm{s})$ & 10 & $16 \mathrm{yr}$ & 38.0 & 28.0 & -10.0 & -26.3 & -62.5 & Yang et al. (2008) \\
\hline Clinton & no till (c-w) & mb till (c-w) & 20 & 22 yr - 1979-2001 & 72.7 & 71.4 & -1.3 & -1.8 & -5.9 & $\begin{array}{l}\text { VandenBygaart and } \\
\text { Kay (2004) }\end{array}$ \\
\hline Clinton & no till (c-w) & mb till (c-w) & 20 & 22 yr - 1979-2001 & 25.7 & 25.3 & -0.4 & -1.6 & -1.8 & $\begin{array}{l}\text { VandenBygaart and } \\
\text { Kay (2004) }\end{array}$ \\
\hline Clinton & no till (c-w) & mb till (c-w) & 20 & 22 yr - 1979-2001 & 57.1 & 54.9 & -2.2 & -3.9 & -10.0 & $\begin{array}{l}\text { VandenBygaart and } \\
\text { Kay (2004) }\end{array}$ \\
\hline Clinton & no till (c-w) & mb till (c-w) & 20 & 22 yr - 1979-2001 & 61.7 & 62.9 & 1.2 & 1.9 & 5.5 & $\begin{array}{l}\text { VandenBygaart and } \\
\text { Kay (2004) }\end{array}$ \\
\hline Clinton & no till (c-w) & mb till (c-w) & 20 & 19 yr - 1981-1999 & 138.1 & 87.9 & -50.2 & -36.3 & -264.1 & Yang and Kay (2001b) \\
\hline Clinton & no till (c-w) & mb till (c-w) & 20 & $19 \mathrm{yr}-1981-1999$ & 99.5 & 86.2 & -13.3 & -13.4 & -69.9 & Yang and Kay (2001b) \\
\hline Clinton & no till (c-w) & mb till (c-w) & 20 & 19 yr - 1981-1999 & 51.5 & 44.1 & -7.5 & -14.5 & -39.4 & Yang and Kay (2001b) \\
\hline Elora & no till (c-s) & $\mathrm{mb}$ till $(\mathrm{c}-\mathrm{s})$ & 20 & $25 \mathrm{yr}-1976-2001$ & 43.7 & 50.4 & 6.7 & 15.3 & 26.8 & Deen and Kataki (2003) \\
\hline Elora & no till (c-s) & mb till (c-s) & 20 & $25 \mathrm{yr}-1976-2001$ & 43.7 & 42.9 & -0.8 & -1.8 & -3.2 & Deen and Kataki (2003) \\
\hline Elora & no till (c-s) & mb till (c-s) & 20 & $25 \mathrm{yr}-1976-2001$ & 43.7 & 49.3 & 5.6 & 12.8 & 22.4 & Deen and Kataki (2003) \\
\hline Elora & no till (c-s) & ch till (c-s) & 20 & $25 \mathrm{yr}-1976-2001$ & 43.7 & 50.0 & 6.3 & 14.4 & 25.2 & Deen and Kataki (2003) \\
\hline Elora & min till (cc) & till $(\mathrm{cc})$ & 20 & $16 \mathrm{yr}-1980-1995$ & 55.7 & 50.4 & -5.2 & -9.4 & -32.6 & $\begin{array}{l}\text { Wanniarachchi et al. } \\
\text { (1999) }\end{array}$ \\
\hline Elora & no till (cc) & mb till $(\mathrm{cc})$ & 20 & $23 \mathrm{yr}$ & 58.0 & 56.1 & -1.9 & -3.3 & -8.3 & Yang et al. (2008) \\
\hline Ridgetown & no till (c-s) & till $(\mathrm{c}-\mathrm{s})$ & 20 & $15 \mathrm{yr}-1991-2006$ & 55.4 & 47.0 & -8.4 & -15.2 & -56.0 & Van Eerd et al. (2014) \\
\hline Ridgetown & no till (cc, no N) & till (cc, no N) & 20 & $11 \mathrm{yr}-1995-2006$ & 63.8 & 46.4 & -17.4 & -27.3 & -116.0 & unpublished \\
\hline Ridgetown & no till (c-s, no N) & till (c-s, no N) & 20 & $11 \mathrm{yr}-1995-2006$ & 55.1 & 52.7 & -2.4 & -4.4 & -16.0 & unpublished \\
\hline Ridgetown & no till (c-s-w, no N) & till (c-s-w, no N) & 20 & $11 \mathrm{yr}-1995-2006$ & 59.3 & 54.7 & -4.6 & -7.8 & -30.7 & unpublished \\
\hline Ridgetown & no till $(\mathrm{cc})$ & till $(\mathrm{cc})$ & 20 & $11 \mathrm{yr}-1995-2006$ & 60.2 & 48.8 & -11.4 & -18.9 & -76.0 & Van Eerd et al. (2014) \\
\hline Ridgetown & no till (c-s) & till (c-s) & 20 & $11 \mathrm{yr}-1995-2006$ & 56.9 & 49.9 & -7.0 & -12.3 & -46.7 & Van Eerd et al. (2014) \\
\hline Ridgetown & no till (c-s-w) & till (c-s-w) & 20 & $11 \mathrm{yr}-1995-2006$ & 66.0 & 53.7 & -12.3 & -18.6 & -82.0 & Van Eerd et al. (2014) \\
\hline Ridgetown & no till (c-s) & ch till (c-s) & 20 & $15 \mathrm{yr}-1991-2006$ & 55.4 & 48.9 & -6.5 & -11.7 & -43.3 & Van Eerd et al. (2014) \\
\hline Woodslee & no till (w-c-s) & mb till (w-c-s) & 20 & $16 \mathrm{yr}-1993-2009$ & 54.3 & 51.6 & -2.7 & -5.0 & -16.9 & Shi et al. (2011) \\
\hline Woodslee & no till (c-s) & mb till (c-s) & 20 & $16 \mathrm{yr}$ & 64.8 & 60.7 & -4.1 & -6.3 & -25.6 & Yang et al. (2008) \\
\hline
\end{tabular}


Tillage systems: no till (control) vs. tillage (treatment)

\begin{tabular}{|c|c|c|c|c|c|c|c|c|c|c|}
\hline Site & Control & Treatment & $\begin{array}{l}\text { Depth } \\
(\mathrm{cm})\end{array}$ & Study duration (yr) & $\begin{array}{c}\text { SOC } \\
\text { Control } \\
\left(\mathrm{Mg} \mathrm{ha}^{-1}\right)\end{array}$ & $\begin{array}{l}\text { SOC TRT } \\
\left(\mathrm{Mg} \mathrm{ha}^{-1}\right)\end{array}$ & $\begin{array}{c}\Delta \mathrm{SOC} \\
\left(\mathrm{Mg} \mathrm{ha}^{-1}\right)\end{array}$ & $\Delta \mathrm{SOC}(\%)$ & $\begin{array}{c}\Delta \mathrm{SOC} \\
\left(\mathrm{g} \mathrm{C} \mathrm{m}^{-2} \mathrm{yr}^{-1}\right)\end{array}$ & Reference \\
\hline Clinton & no till (c-w) & mb till (c-w) & 30 & $22 \mathrm{yr}-1979-2001$ & 80.8 & 82.4 & 1.6 & 2.0 & 7.3 & $\begin{array}{l}\text { VandenBygaart and } \\
\text { Kay (2004) }\end{array}$ \\
\hline Clinton & no till (c-w) & mb till (c-w) & 30 & $22 \mathrm{yr}-1979-2001$ & 32.8 & 31.0 & -1.8 & -5.5 & -8.2 & $\begin{array}{l}\text { VandenBygaart and } \\
\text { Kay (2004) }\end{array}$ \\
\hline Clinton & no till (c-w) & mb till (c-w) & 30 & $22 \mathrm{yr}-1979-2001$ & 78.9 & 75.2 & -3.7 & -4.7 & -16.8 & $\begin{array}{l}\text { VandenBygaart and } \\
\text { Kay (2004) }\end{array}$ \\
\hline Clinton & no till (c-w) & mb till (c-w) & 30 & 22 yr - 1979-2001 & 93.1 & 93.9 & 0.8 & 0.9 & 3.6 & $\begin{array}{l}\text { VandenBygaart and } \\
\text { Kay (2004) }\end{array}$ \\
\hline Elora & no till & till & 30 & $25 \mathrm{yr}-1980-2005$ & & & 1.5 & & 6.0 & $\begin{array}{l}\text { VandenBygaart et al. } \\
(2010)\end{array}$ \\
\hline Woodslee & no till (w-c-s) & mb till (w-c-s) & 30 & $16 \mathrm{yr}-1993-2009$ & 72.5 & 71.7 & -0.8 & -1.1 & -5.0 & Shi et al. (2011) \\
\hline Woodslee & no till & till & 30 & $12 \mathrm{yr}-1996-2005$ & & & 1.5 & & 12.5 & $\begin{array}{l}\text { Vandenbygaart et al. } \\
(2010)\end{array}$ \\
\hline Clinton & no till (c-w) & $\mathrm{mb}$ till $(\mathrm{c}-\mathrm{w})$ & 40 & $19 \mathrm{yr}-1981-1999$ & 185.8 & 110.8 & -75.0 & -40.4 & -394.7 & Yang and Kay (2001b) \\
\hline Clinton & no till (c-w) & $\mathrm{mb}$ till $(\mathrm{c}-\mathrm{w})$ & 40 & $19 \mathrm{yr}-1981-1999$ & 136.6 & 125.7 & -10.9 & -8.0 & -57.3 & Yang and Kay (2001b) \\
\hline Clinton & no till (c-w) & $\mathrm{mb}$ till (c-w) & 40 & $19 \mathrm{yr}-1981-1999$ & 66.7 & 65.4 & -1.3 & -2.0 & -7.1 & Yang and Kay (2001b) \\
\hline Elora & no till (c-s) & $\mathrm{mb}$ till (c-s) & 40 & $25 \mathrm{yr}-1976-2001$ & 78.2 & 88.3 & 10.0 & 12.8 & 40.1 & Deen and Kataki (2003) \\
\hline Elora & no till (c-s) & $\mathrm{mb}$ till $(\mathrm{c}-\mathrm{s})$ & 40 & $25 \mathrm{yr}-1976-2001$ & 78.2 & 90.0 & 11.8 & 15.1 & 47.3 & Deen and Kataki (2003) \\
\hline Elora & no till (c-s) & mb till (c-s) & 40 & $25 \mathrm{yr}-1976-2001$ & 78.2 & 85.1 & 6.9 & 8.8 & 27.6 & Deen and Kataki (2003) \\
\hline Elora & no till (c-s) & ch till (c-s) & 40 & $25 \mathrm{yr}-1976-2001$ & 78.2 & 81.4 & 3.2 & 4.1 & 12.8 & Deen and Kataki (2003) \\
\hline Elora & min till $(\mathrm{cc})$ & till (cc) & 40 & $16 \mathrm{yr}-1980-1995$ & 67.7 & 67.7 & 0.0 & 0.0 & 0.0 & $\begin{array}{l}\text { Wanniarachchi et al. } \\
\text { (1999) }\end{array}$ \\
\hline Elora & no till $(\mathrm{cc})$ & $\mathrm{mb}$ till $(\mathrm{cc})$ & 40 & $23 \mathrm{yr}$ & 81.6 & 80.1 & -1.5 & -1.8 & -6.5 & Yang et al. (2008) \\
\hline Ridgetown & no till (c-s) & till $(\mathrm{c}-\mathrm{s})$ & 40 & $15 \mathrm{yr}-1991-2006$ & 90.7 & 73.7 & -17.0 & -18.7 & -113.3 & Van Eerd et al. (2014) \\
\hline Ridgetown & no till (cc, no N) & till (cc, no N) & 40 & $11 \mathrm{yr}-1995-2006$ & 107.8 & 73.8 & -34.0 & -31.5 & -226.7 & unpublished \\
\hline Ridgetown & no till (c-s, no N) & till $(\mathrm{c}-\mathrm{s}$, no $\mathrm{N})$ & 40 & $11 \mathrm{yr}-1995-2006$ & 84.7 & 81.5 & -3.2 & -3.8 & -21.3 & unpublished \\
\hline Ridgetown & no till (c-s-w, no N) & till (c-s-w, no N) & 40 & $11 \mathrm{yr}-1995-2006$ & 100.5 & 89.3 & -11.2 & -11.1 & -74.7 & unpublished \\
\hline Ridgetown & no till $(\mathrm{cc})$ & till $(\mathrm{cc})$ & 40 & $11 \mathrm{yr}-1995-2006$ & 104.2 & 79.3 & -24.9 & -23.9 & -166.0 & Van Eerd et al. (2014) \\
\hline Ridgetown & no till (c-s) & till $(\mathrm{c}-\mathrm{s})$ & 40 & $11 \mathrm{yr}-1995-2006$ & 96.8 & 80.9 & -15.9 & -16.4 & -106.0 & Van Eerd et al. (2014) \\
\hline Ridgetown & no till (c-s-w) & till (c-s-w) & 40 & $11 \mathrm{yr}-1995-2006$ & 100.6 & 87.7 & -12.9 & -12.8 & -86.0 & Van Eerd et al. (2014) \\
\hline Ridgetown & no till (c-s) & ch till (c-s) & 40 & $15 \mathrm{yr}-1991-2006$ & 90.7 & 77.6 & -13.1 & -14.4 & -87.3 & Van Eerd et al. (2014) \\
\hline Woodslee & no till (c-s) & mb till (c-s) & 40 & $16 \mathrm{yr}$ & 98.9 & 98.0 & -0.9 & -0.9 & -5.6 & Yang et al. (2008) \\
\hline Dresden & before till (c-s-w) & after mb till & 45 & $15 \mathrm{yr}-1985-2000$ & 56.0 & 62.1 & 6.1 & 10.9 & 40.7 & $\begin{array}{l}\text { VandenBygaart et al. } \\
(2002,2003)\end{array}$ \\
\hline Dresden & before till (c-s-w) & after mb till & 45 & $15 \mathrm{yr}-1985-2000$ & 53.3 & 55.3 & 2.0 & 3.8 & 13.3 & $\begin{array}{l}\text { VandenBygaart et al. } \\
(2002,2003)\end{array}$ \\
\hline Dresden & before till (c-s-w) & after mb till & 45 & $15 \mathrm{yr}-1985-2000$ & 40.8 & 35.7 & -5.1 & -12.5 & -34.0 & $\begin{array}{l}\text { VandenBygaart et al. } \\
(2002,2003)\end{array}$ \\
\hline Dresden & before till (c-s-w) & after mb till & 45 & $15 \mathrm{yr}-1985-2000$ & 36.3 & 28.0 & -8.3 & -22.9 & -55.3 & $\begin{array}{l}\text { VandenBygaart et al. } \\
(2002,2003)\end{array}$ \\
\hline Paris & before till (c-s-w) & after mb till & 45 & $15 \mathrm{yr}-1985-2000$ & 41.1 & 42.4 & 1.3 & 3.2 & 8.7 & $\begin{array}{l}\text { VandenBygaart et al. } \\
(2002,2003)\end{array}$ \\
\hline Paris & before till (c-s-w) & after mb till & 45 & $15 \mathrm{yr}-1985-2000$ & 46.7 & 59.0 & 12.3 & 26.3 & 82.0 & $\begin{array}{l}\text { VandenBygaart et al. } \\
(2002,2003)\end{array}$ \\
\hline Paris & before till (c-s-w) & after mb till & 45 & $15 \mathrm{yr}-1985-2000$ & 37.7 & 39.2 & 1.5 & 4.0 & 10.0 & $\begin{array}{l}\text { VandenBygaart et al. } \\
(2002,2003)\end{array}$ \\
\hline Paris & before till (c-s-w) & after mb till & 45 & $15 \mathrm{yr}-1985-2000$ & 36.0 & 36.9 & 0.9 & 2.5 & 6.0 & $\begin{array}{l}\text { VandenBygaart et al. } \\
(2002,2003)\end{array}$ \\
\hline Paris & before till (c-s-w) & after mb till & 45 & $15 \mathrm{yr}-1985-2000$ & 20.5 & 16.5 & -4.0 & -19.5 & -26.7 & $\begin{array}{l}\text { VandenBygaart et al. } \\
(2002,2003)\end{array}$ \\
\hline
\end{tabular}


Tillage systems: no till (control) vs. tillage (treatment)

\begin{tabular}{|c|c|c|c|c|c|c|c|c|c|c|}
\hline Site & Control & Treatment & $\begin{array}{l}\text { Depth } \\
(\mathrm{cm})\end{array}$ & Study duration (yr) & $\begin{array}{c}\text { SOC } \\
\text { Control } \\
\left(\mathrm{Mg} \mathrm{ha}^{-1}\right)\end{array}$ & $\begin{array}{l}\text { SOC TRT } \\
\left(\mathrm{Mg} \mathrm{ha}^{-1}\right)\end{array}$ & $\begin{array}{c}\Delta \mathrm{SOC} \\
\left(\mathrm{Mg} \mathrm{ha}^{-1}\right)\end{array}$ & $\Delta \mathrm{SOC}(\%)$ & $\begin{array}{c}\Delta \mathrm{SOC} \\
\left(\mathrm{g} \mathrm{C} \mathrm{m}^{-2} \mathrm{yr}^{-1}\right)\end{array}$ & Reference \\
\hline Paris & before till (c-s-w) & after mb till & 45 & $15 \mathrm{yr}-1985-2000$ & 42.4 & 41.5 & -0.9 & -2.1 & -6.0 & $\begin{array}{l}\text { VandenBygaart et al. } \\
(2002,2003)\end{array}$ \\
\hline Paris & before till (c-s-w) & after $m b$ till & 45 & $15 \mathrm{yr}-1985-2000$ & 33.1 & 35.0 & 1.9 & 5.7 & 12.7 & $\begin{array}{l}\text { VandenBygaart et al. } \\
(2002,2003)\end{array}$ \\
\hline Paris & before till (c-s-w) & after mb till & 45 & $15 \mathrm{yr}-1985-2000$ & 30.0 & 29.8 & -0.2 & -0.7 & -1.3 & $\begin{array}{l}\text { Vanden Bygaart et al. } \\
(2002,2003)\end{array}$ \\
\hline Paris & before till (c-s-w) & after $\mathrm{mb}$ till & 45 & $15 \mathrm{yr}-1985-2000$ & 36.4 & 38.6 & 2.2 & 6.0 & 14.7 & $\begin{array}{l}\text { Vanden Bygaart et al. } \\
(2002,2003)\end{array}$ \\
\hline Paris & before till (c-s-w) & after $m b$ till & 45 & $15 \mathrm{yr}-1985-2000$ & 21.1 & 8.6 & -12.5 & -59.2 & -83.3 & $\begin{array}{l}\text { VandenBygaart et al. } \\
(2002,2003)\end{array}$ \\
\hline Paris & before till (c-s-w) & after mb till & 45 & $15 \mathrm{yr}-1985-2000$ & 49.5 & 59.0 & 9.5 & 19.2 & 63.3 & $\begin{array}{l}\text { Vanden Bygaart et al. } \\
(2002,2003)\end{array}$ \\
\hline Paris & before till (c-s-w) & after $m b$ till & 45 & $15 \mathrm{yr}-1985-2000$ & 31.3 & 23.8 & -7.5 & -24.0 & -50.0 & $\begin{array}{l}\text { VandenBygaart et al. } \\
(2002,2003)\end{array}$ \\
\hline Paris & before till (c-s-w) & after $m b$ till & 45 & $15 \mathrm{yr}-1985-2000$ & 24.8 & 21.5 & -3.3 & -13.3 & -22.0 & $\begin{array}{l}\text { VandenBygaart et al. } \\
(2002,2003)\end{array}$ \\
\hline Paris & before till (c-s-w) & after mb till & 45 & $15 \mathrm{yr}-1985-2000$ & 33.3 & 34.1 & 0.8 & 2.4 & 5.3 & $\begin{array}{l}\text { Vanden Bygaart et al. } \\
(2002,2003)\end{array}$ \\
\hline Paris & before till (c-s-w) & after mb till & 45 & $15 \mathrm{yr}-1985-2000$ & 45.9 & 48.4 & 2.5 & 5.4 & 16.7 & $\begin{array}{l}\text { VandenBygaart et al. } \\
(2002,2003)\end{array}$ \\
\hline Strathroy & before till (c-s-w) & after mb till & 45 & $15 \mathrm{yr}-1985-2000$ & 61.6 & 81.8 & 20.2 & 32.8 & 134.7 & $\begin{array}{l}\text { VandenBygaart et al. } \\
(2002,2003)\end{array}$ \\
\hline Strathroy & before till (c-s-w) & after mb till & 45 & $15 \mathrm{yr}-1985-2000$ & 47.7 & 48.1 & 0.4 & 0.8 & 2.7 & $\begin{array}{l}\text { Vanden Bygaart et al. } \\
(2002,2003)\end{array}$ \\
\hline Strathroy & before till (c-s-w) & after mb till & 45 & $15 \mathrm{yr}-1985-2000$ & 43.8 & 48.4 & 4.6 & 10.5 & 30.7 & $\begin{array}{l}\text { Vanden Bygaart et al. } \\
(2002,2003)\end{array}$ \\
\hline Strathroy & before till (c-s-w) & after mb till & 45 & $15 \mathrm{yr}-1985-2000$ & 47.0 & 56.1 & 9.1 & 19.4 & 60.7 & $\begin{array}{l}\text { VandenBygaart et al. } \\
(2002,2003)\end{array}$ \\
\hline Strathroy & before till (c-s-w) & after mb till & 45 & $15 \mathrm{yr}-1985-2000$ & 38.9 & 49.0 & 10.1 & 26.0 & 67.3 & $\begin{array}{l}\text { VandenBygaart et al. } \\
(2002,2003)\end{array}$ \\
\hline Strathroy & before till (c-s-w) & after mb till & 45 & $15 \mathrm{yr}-1985-2000$ & 46.6 & 60.9 & 14.3 & 30.7 & 95.3 & $\begin{array}{l}\text { Vanden Bygaart et al. } \\
(2002,2003)\end{array}$ \\
\hline Strathroy & before till (c-s-w) & after mb till & 45 & $15 \mathrm{yr}-1985-2000$ & 41.1 & 38.1 & -3.0 & -7.3 & -20.0 & $\begin{array}{l}\text { VandenBygaart et al. } \\
(2002,2003)\end{array}$ \\
\hline Strathroy & before till (c-s-w) & after mb till & 45 & $15 \mathrm{yr}-1985-2000$ & 44.1 & 48.0 & 3.9 & 8.8 & 26.0 & $\begin{array}{l}\text { VandenBygaart et al. } \\
(2002,2003)\end{array}$ \\
\hline Strathroy & before till (c-s-w) & after mb till & 45 & $15 \mathrm{yr}-1985-2000$ & 44.2 & 55.1 & 10.9 & 24.7 & 72.7 & $\begin{array}{l}\text { VandenBygaart et al. } \\
(2002,2003)\end{array}$ \\
\hline Throndale & before till (c-s-w) & after mb till & 45 & $15 \mathrm{yr}-1985-2000$ & 75.1 & 77.2 & 2.1 & 2.8 & 14.0 & $\begin{array}{l}\text { VandenBygaart et al. } \\
(2002,2003)\end{array}$ \\
\hline Throndale & before till (c-s-w) & after mb till & 45 & $15 \mathrm{yr}-1985-2000$ & 76.0 & 88.7 & 12.7 & 16.7 & 84.7 & $\begin{array}{l}\text { VandenBygaart et al. } \\
(2002,2003)\end{array}$ \\
\hline Throndale & before till (c-s-w) & after mb till & 45 & $15 \mathrm{yr}-1985-2000$ & 48.8 & 37.2 & -11.6 & -23.8 & -77.3 & $\begin{array}{l}\text { VandenBygaart et al. } \\
(2002,2003)\end{array}$ \\
\hline Throndale & before till (c-s-w) & after mb till & 45 & $15 \mathrm{yr}-1985-2000$ & 84.1 & 86.1 & 2.0 & 2.4 & 13.3 & $\begin{array}{l}\text { VandenBygaart et al. } \\
(2002,2003)\end{array}$ \\
\hline Throndale & before till (c-s-w) & after mb till & 45 & $15 \mathrm{yr}-1985-2000$ & 62.7 & 66.5 & 3.8 & 6.1 & 25.3 & $\begin{array}{l}\text { VandenBygaart et al. } \\
(2002,2003)\end{array}$ \\
\hline
\end{tabular}


Tillage systems: no till (control) vs. tillage (treatment)

\begin{tabular}{|c|c|c|c|c|c|c|c|c|c|c|}
\hline Site & Control & Treatment & $\begin{array}{l}\text { Depth } \\
(\mathrm{cm})\end{array}$ & Study duration (yr) & $\begin{array}{c}\text { SOC } \\
\text { Control } \\
\left(\mathrm{Mg} \mathrm{ha}^{-1}\right)\end{array}$ & $\begin{array}{l}\text { SOC TRT } \\
\left(\mathrm{Mg} \mathrm{ha}^{-1}\right)\end{array}$ & $\begin{array}{c}\Delta \mathrm{SOC} \\
\left(\mathrm{Mg} \mathrm{ha}^{-1}\right)\end{array}$ & $\Delta \mathrm{SOC}(\%)$ & $\begin{array}{c}\Delta \mathrm{SOC} \\
\left(\mathrm{g} \mathrm{C} \mathrm{m}^{-2} \mathrm{yr}^{-1}\right)\end{array}$ & Reference \\
\hline Throndale & before till (c-s-w) & after mb till & 45 & $15 \mathrm{yr}-1985-2000$ & 39.8 & 17.8 & -22.0 & -55.3 & -146.7 & $\begin{array}{l}\text { VandenBygaart et al. } \\
(2002,2003)\end{array}$ \\
\hline Throndale & before till (c-s-w) & after $m b$ till & 45 & $15 \mathrm{yr}-1985-2000$ & 79.1 & 117.3 & 38.2 & 48.3 & 254.7 & $\begin{array}{l}\text { VandenBygaart et al. } \\
(2002,2003)\end{array}$ \\
\hline Throndale & before till (c-s-w) & after mb till & 45 & $15 \mathrm{yr}-1985-2000$ & 88.2 & 108.7 & 20.5 & 23.2 & 136.7 & $\begin{array}{l}\text { VandenBygaart et al. } \\
(2002,2003)\end{array}$ \\
\hline Throndale & before till (c-s-w) & after $\mathrm{mb}$ till & 45 & $15 \mathrm{yr}-1985-2000$ & 47.4 & 45.5 & -1.9 & -4.0 & -12.7 & $\begin{array}{l}\text { VandenBygaart et al. } \\
(2002,2003)\end{array}$ \\
\hline Clinton & no till (c-w) & mb till (c-w) & 50 & 19 yr - 1981-1999 & 198.4 & 117.8 & -80.6 & -40.6 & -424.3 & Yang and Kay (2001b) \\
\hline Clinton & no till (c-w) & $\mathrm{mb}$ till (c-w) & 50 & $19 \mathrm{yr}-1981-1999$ & 151.2 & 148.6 & -2.6 & -1.7 & -13.6 & Yang and Kay (2001b) \\
\hline Clinton & no till (c-w) & $\mathrm{mb}$ till (c-w) & 50 & $19 \mathrm{yr}-1981-1999$ & 84.2 & 79.9 & -4.3 & -5.1 & -22.6 & Yang and Kay (2001b) \\
\hline Elora & min till (cc) & till $(\mathrm{cc})$ & 50 & $16 \mathrm{yr}-1980-1995$ & 71.7 & 72.2 & 0.5 & 0.7 & 3.1 & $\begin{array}{l}\text { Wanniarachchi et al. } \\
\text { (1999) }\end{array}$ \\
\hline Elora & no till (cc) & $\mathrm{mb}$ till $(\mathrm{cc})$ & 50 & $23 \mathrm{yr}$ & 87.8 & 85.3 & -2.5 & -2.8 & -10.9 & Yang et al. (2008) \\
\hline Woodslee & no till (c-s) & mb till (c-s) & 50 & $16 \mathrm{yr}$ & 105.9 & 106.4 & 0.5 & 0.5 & 3.1 & Yang et al. (2008) \\
\hline Elora & no till (c-s) & $\mathrm{mb}$ till $(\mathrm{c}-\mathrm{s})$ & 60 & $25 \mathrm{yr}-1976-2001$ & 103.7 & 110.2 & 6.5 & 6.2 & 25.9 & Deen and Kataki (2003) \\
\hline Elora & no till (c-s) & mb till (c-s) & 60 & $25 \mathrm{yr}-1976-2001$ & 103.7 & 120.9 & 17.1 & 16.5 & 68.6 & Deen and Kataki (2003) \\
\hline Elora & no till (c-s) & mb till (c-s) & 60 & $25 \mathrm{yr}-1976-2001$ & 103.7 & 99.1 & -4.6 & -4.4 & -18.4 & Deen and Kataki (2003) \\
\hline Elora & no till (c-s) & ch till $(\mathrm{c}-\mathrm{s})$ & 60 & $25 \mathrm{yr}-1976-2001$ & 103.7 & 94.8 & -8.9 & -8.6 & -35.5 & Deen and Kataki (2003) \\
\hline Ridgetown & no till (c-s) & till (c-s) & 120 & $15 \mathrm{yr}-1991-2006$ & 190.9 & 169.8 & -21.1 & -11.1 & -140.7 & Van Eerd et al. (2014) \\
\hline Ridgetown & no till $(\mathrm{cc}$, no $\mathrm{N})$ & till $(\mathrm{cc}$, no $\mathrm{N})$ & 120 & $11 \mathrm{yr}-1995-2006$ & 231.3 & 161.7 & -69.6 & -30.1 & -464.0 & Van Eerd et al. (2014) \\
\hline Ridgetown & no till (c-s, no N) & till $(\mathrm{c}-\mathrm{s}$, no N) & 120 & $11 \mathrm{yr}-1995-2006$ & 196.0 & 169.7 & -26.3 & -13.4 & -175.3 & Van Eerd et al. (2014) \\
\hline Ridgetown & no till (c-s-w, no N) & till (c-s-w, no N) & 120 & $11 \mathrm{yr}-1995-2006$ & 212.9 & 186.3 & -26.6 & -12.5 & -177.3 & Van Eerd et al. (2014) \\
\hline Ridgetown & no till $(\mathrm{cc})$ & till $(\mathrm{cc})$ & 120 & $11 \mathrm{yr}-1995-2006$ & 221.2 & 179.0 & -42.2 & -19.1 & -281.3 & Van Eerd et al. (2014) \\
\hline Ridgetown & no till (c-s) & till $(\mathrm{c}-\mathrm{s})$ & 120 & $11 \mathrm{yr}-1995-2006$ & 198.3 & 243.7 & 45.4 & 22.9 & 302.7 & Van Eerd et al. (2014) \\
\hline Ridgetown & no till (c-s-w) & till (c-s-w) & 120 & $11 \mathrm{yr}-1995-2006$ & 213.4 & 170.3 & -43.1 & -20.2 & -287.3 & Van Eerd et al. (2014) \\
\hline Ridgetown & no till (c-s) & ch till (c-s) & 120 & $15 \mathrm{yr}-1991-2006$ & 190.9 & 178.8 & -12.1 & -6.3 & -80.7 & Van Eerd et al. (2014) \\
\hline \multicolumn{11}{|c|}{ Fertilizer Systems: no $N$ fertilizer (control) vs. $N$ fertilizer (treatment) } \\
\hline Site & Control & Treatment & $\begin{array}{l}\text { Depth } \\
(\mathrm{cm})\end{array}$ & Study duration (yr) & $\begin{array}{c}\text { SOC } \\
\text { Control } \\
\left(\mathrm{Mg} \mathrm{ha}^{-1}\right)\end{array}$ & $\begin{array}{l}\text { SOC TRT } \\
\left(\mathrm{Mg} \mathrm{ha}^{-1}\right)\end{array}$ & $\begin{array}{c}\Delta \mathrm{SOC} \\
\left(\mathrm{Mg} \mathrm{ha}^{-1}\right)\end{array}$ & $\Delta \mathrm{SOC}(\%)$ & $\begin{array}{c}\Delta \mathrm{SOC} \\
\left(\mathrm{g} \mathrm{C} \mathrm{m}^{-2} \mathrm{yr}^{-1}\right)\end{array}$ & Reference \\
\hline Ridgetown & no $\mathrm{N}$ (cc, till) & $\mathrm{N}(\mathrm{cc}$, till $)$ & 10 & $11 \mathrm{yr}-1995-2006$ & 23.4 & 30.8 & 7.4 & 31.6 & 49.3 & unpublished \\
\hline Ridgetown & no $N(\mathrm{c}-\mathrm{s}$, till $)$ & $\mathrm{N}(\mathrm{c}-\mathrm{s}$, till $)$ & 10 & $11 \mathrm{yr}-1995-2006$ & 25.8 & 30.6 & 4.8 & 18.6 & 32.0 & unpublished \\
\hline Ridgetown & no N (c-s-w, till) & $\mathrm{N}(\mathrm{c}-\mathrm{s}-\mathrm{w}$, till $)$ & 10 & $11 \mathrm{yr}-1995-2006$ & 27.4 & 35.4 & 8.0 & 29.2 & 53.3 & unpublished \\
\hline Woodslee & no $\mathrm{N}(\mathrm{cc}$, till $)$ & $\mathrm{N}(\mathrm{cc}$, till $)$ & 10 & $32 \mathrm{yr}-1959-1991$ & 19.5 & 21.8 & 2.3 & 11.8 & 7.2 & Gregorich et al. (1996) \\
\hline Woodslee & no N (cc, till) & $\mathrm{N}(\mathrm{cc}$, till $)$ & 10 & $35 \mathrm{yr}-1959-1994$ & 23.1 & 26.9 & 3.8 & 16.5 & 10.9 & Gregorich et al. (2001) \\
\hline Woodslee & no N (c-o-a-a, till) & $\mathrm{N}(\mathrm{c}-\mathrm{o}-\mathrm{a}-\mathrm{a}$, till $)$ & 10 & $35 \mathrm{yr}-1959-1994$ & 27.8 & 27.1 & -0.7 & -2.5 & -2.0 & Gregorich et al. (2001) \\
\hline Ridgetown & no $N(\mathrm{cc}$, till $)$ & $\mathrm{N}(\mathrm{cc}, \mathrm{till})$ & 20 & $11 \mathrm{yr}-1995-2006$ & 46.4 & 60.2 & 13.8 & 29.7 & 92.0 & unpublished \\
\hline Ridgetown & no N (c-s, till) & $\mathrm{N}(\mathrm{c}-\mathrm{s}$, till $)$ & 20 & $11 \mathrm{yr}-1995-2006$ & 52.7 & 56.9 & 4.2 & 8.0 & 28.0 & unpublished \\
\hline Ridgetown & no $\mathrm{N}$ (c-s-w, till) & $\mathrm{N}(\mathrm{c}-\mathrm{s}-\mathrm{w}$, till $)$ & 20 & $11 \mathrm{yr}-1995-2006$ & 54.7 & 66.0 & 11.3 & 20.7 & 75.3 & unpublished \\
\hline Woodslee & no N (cc, till $)$ & $\mathrm{N}(\mathrm{cc}$, till $)$ & 20 & $32 \mathrm{yr}-1959-1991$ & 39.3 & 43.5 & 4.2 & 10.7 & 13.1 & Gregorich et al. (1996) \\
\hline Woodslee & no $\mathrm{N}(\mathrm{cc}$, till $)$ & $\mathrm{N}(\mathrm{cc}, \mathrm{till})$ & 20 & $35 \mathrm{yr}-1959-1994$ & 51.4 & 55.6 & 4.2 & 8.2 & 12.0 & Gregorich et al. (2001) \\
\hline Woodslee & no N (c-o-a-a, till) & $N(c-o-a-a$, till $)$ & 20 & $35 \mathrm{yr}-1959-1994$ & 56.9 & 55.9 & -1.0 & -1.8 & -2.9 & Gregorich et al. (2001) \\
\hline Ridgetown & no $N(\mathrm{cc}$, till $)$ & $\mathrm{N}(\mathrm{cc}$, till $)$ & 40 & $11 \mathrm{yr}-1995-2006$ & 73.8 & 104.2 & 30.4 & 41.2 & 202.7 & unpublished \\
\hline Ridgetown & no $N(c-s$, till $)$ & $\mathrm{N}(\mathrm{c}-\mathrm{s}$, till $)$ & 40 & $11 \mathrm{yr}-1995-2006$ & 81.5 & 96.8 & 15.3 & 18.8 & 102.0 & unpublished \\
\hline
\end{tabular}


in the Canadian Prairies available at URL: http://www. pedosphere.ca/CanEduRes/crop_rotations/bibliography. $\mathrm{cfm}$. Opportunities for a more coordinated approach to long-term research could be implemented across Canada, similar to the newly formed USDA Longterm Agro-Ecosystem Research (LTAR) Network for Agriculture (Walbridge and Shafer 2011). Establishing a long-term research network in Ontario may help maintain existing or newly established long-term trials, increase collaboration among researchers, facilitate the use of long-term trials as platforms for testing new hypotheses, and support consistency of data collection among long-term trials. The inclusion of newer $(<10 \mathrm{yr})$ long-term trials in the inventory (Table 2) may stimulate awareness, collaboration, and future research objectives. After all, the success of long-term research depends on collaboration, and the chief justification for its existence is the potential for answering questions not yet envisioned (Janzen 1995).

\section{Long-term Agricultural Land Use}

Non-cropped forest soils had $27.6 \pm 3.46 \%(32.7 \pm 4.46$ $\mathrm{Mg} \mathrm{C} \mathrm{ha}{ }^{-1}$ ) higher SOC than long-term agricultural cropping (Table 3). A previous meta-analysis showed similar reductions of SOC $24 \pm 6 \%$ and $22 \pm 9 \%$ in longterm cropped soils compared with nearby forested soil across Canada and in eastern Canada, respectively (VandenBygaart et al. 2003). The observed lower SOC in agricultural compared with forest soil may be attributed to reduced $\mathrm{C}$ inputs, increased rates of organic matter decay (Ellert and Gregorich 1996) and/or $\mathrm{CO}_{2}$ efflux. It was previously reported that SOC in cultivated soils in Quebec and Prince Edward Island was lower than the forested area by $35 \%$ in Podzolic soils; however, cultivation increased SOC by $25 \%$ in Brunisolic and Gleysolic soils (Carter et al. 1998). In Ontario, there was more variability in SOC within forest soil compared with agricultural soil, which was likely due to soil homogenization with tree removal and subsequent land levelling and tillage on agricultural land.

Differences in SOC between adjacent agricultural and forested soil varied across sites, from $-3.0 \%$ at Ottawa to $-68 \%$ at Edwards (Table 3). The sites with the greatest difference in SOC (> unweighted mean) between adjacent agricultural and forested soil were Edwards, Elora, Exeter, Plainfield, Ste. Anne, Winchester, and Woodslee (Table 3), of which some common characteristics included soil great groups of mainly the Gray Brown or Luvisol and Humic Gleysol, soil textures of silty loam or silty clay, and the production of corn. Perhaps new site-specific models should be developed to better predict the SOC loss from different soil groups and areas within Ontario. This information may add critical knowledge for soil $\mathrm{C}$ processes in differing landscapes, climatic environments, soil types/textures, and management practices in Ontario.

According to the Intergovernmental Panel on Climate Change (IPCC 1997) model: Soil $C$ managed $=$ Soil $C$ 


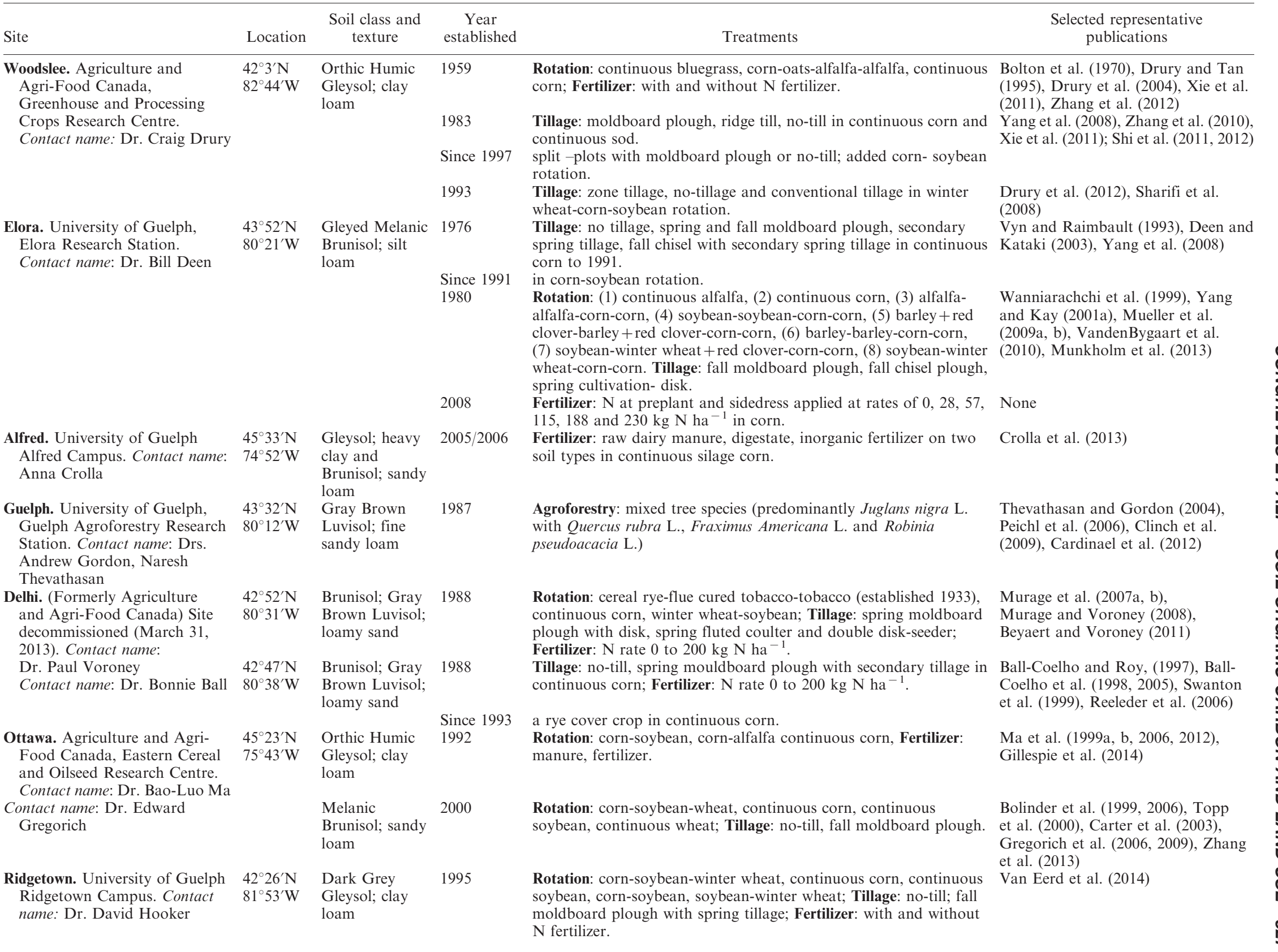




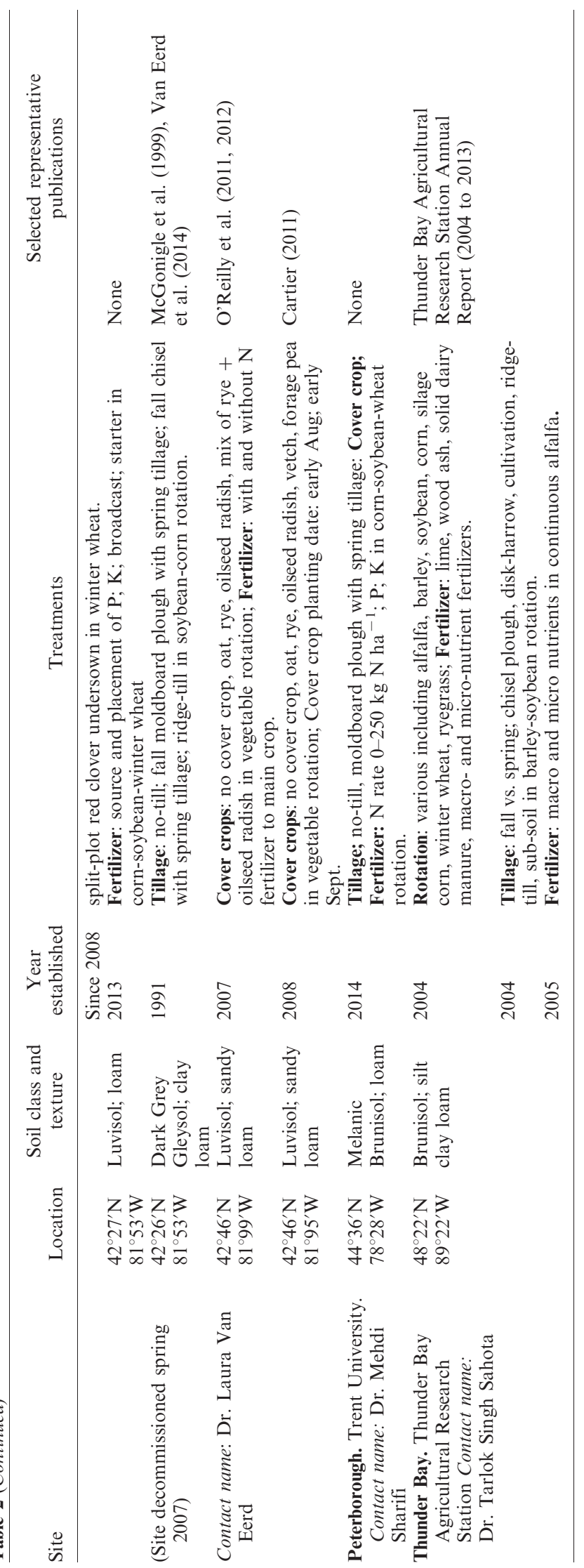

Table 3. Meta-analysis of the effect of forest (control) conversion to long-term cropland (treatment) on the mean change in soil organic $\mathrm{C}$ in the 0 - to $30-\mathrm{cm}$ or 0 - to $40-\mathrm{cm}$ depth at 17 sites in Ontario

\begin{tabular}{|c|c|c|}
\hline \multirow[b]{2}{*}{ Site } & \multicolumn{2}{|c|}{ Mean change in soil organic $\mathrm{C}^{\mathbf{z}}$} \\
\hline & $\begin{array}{c}\text { Net }\left(\mathrm{Mg} \mathrm{ha}^{-1}\right) \\
\quad(n=27)\end{array}$ & $\begin{array}{l}\text { Relative }(\%) \\
\quad(n=28)\end{array}$ \\
\hline Bond Head ${ }^{y}$ & $-22.0 a b$ & $-24.3 a b c d$ \\
\hline C Blondeau & $-27.7 a b c$ & $-20.3 a b c$ \\
\hline Delhi & $-30.4 a b c$ & $-55.3 c d$ \\
\hline Edwards & $-91.1 c$ & $-68.2 d$ \\
\hline Elora & $-42.6 a b c$ & $-40.7 b c d$ \\
\hline Exeter & $-36.1 a b c$ & $-37.9 a b c d$ \\
\hline Fonthill & $-18.7 a b$ & $-23.5 a b c d$ \\
\hline Highgate & $-15.1 a b$ & $-7.7 a b$ \\
\hline Kapuskasking & $-3.30 a b$ & $-4.2 a b$ \\
\hline Kemptville & $-24.9 a b$ & $-30.5 a b c d$ \\
\hline Ottawa & $-8.10 a$ & $-3.0 a$ \\
\hline Panmure & $-20.0 a b$ & $-23.6 a b c d$ \\
\hline Plainfield & $-42.4 a b c$ & $-40.3 b c d$ \\
\hline Ste. Anne & $-37.0 a b c$ & $-34.2 a b c d$ \\
\hline Vineland & $-16.4 a b$ & $-29.0 a b c d$ \\
\hline Winchester & $-43.8 a b c$ & $-34.8 b c d$ \\
\hline Woodslee & $-45.1 b c$ & $-30.6 b c$ \\
\hline Mean & -32.7 & -27.6 \\
\hline $95 \%$ confidence interval & -41.5 to -24.0 & -34.4 to -20.8 \\
\hline Standard error & 4.46 & 3.46 \\
\hline
\end{tabular}

${ }^{\mathrm{z}}$ Negative values indicate lower soil organic $\mathrm{C}$ with cropland compared with forest soil.

${ }^{\mathbf{y}}$ Publications include: Coote and Ramsey (1983), Gregorich et al. (1995), Ellert and Gregorich (1996), Wanniarachchi et al. (1999), Gregorich et al. (2001).

$a-d$ In each column, means followed by different letters indicate a significant difference among sites with a LSD test at $P<0.05$.

native $\times$ Base factor $\times$ Tillage factor $\times$ Input factors, agricultural production reduces SOC by approximately $30 \%$ compared with forested soils, similar to our metaanalysis findings (Table 3). Although there were 17 sites in the meta-analysis, land conversion in northern Ontario was under-represented because only one northern site (Kapuskasing) was included (Fig. 1). Predictions of SOC loss for areas with agricultural potential in northern Ontario would be useful to estimate the future impact of land use transition that may occur with climate change.

\section{Long-term Agricultural Management Practices on SOC}

\section{Tillage}

The meta-analysis showed long-term NT practices had higher SOC in surface layers $(0-10 \mathrm{~cm})$ compared with CT regimes (Table 4), which was consistent with other studies of eastern Canadian soils (Angers et al. 1997; VandenBygaart et al. 2002; Halpern et al. 2010) and elsewhere (Franchini et al. 2007; Fuentes et al. 2009; Deubel et al. 2011). Conventional tillage had $41 \mathrm{~g} \mathrm{C} \mathrm{m}^{-2}$ $\mathrm{yr}^{-1}$ less SOC than NT in the shallow soil depth ranges (0-10 $\mathrm{cm}$ and $0-20 \mathrm{~cm}$ depths) (Table 4), a trend also observed in eastern Canada (Angers et al. 1997; Carter 2005; VandenBygaart et al. 2011) and elsewhere (West 


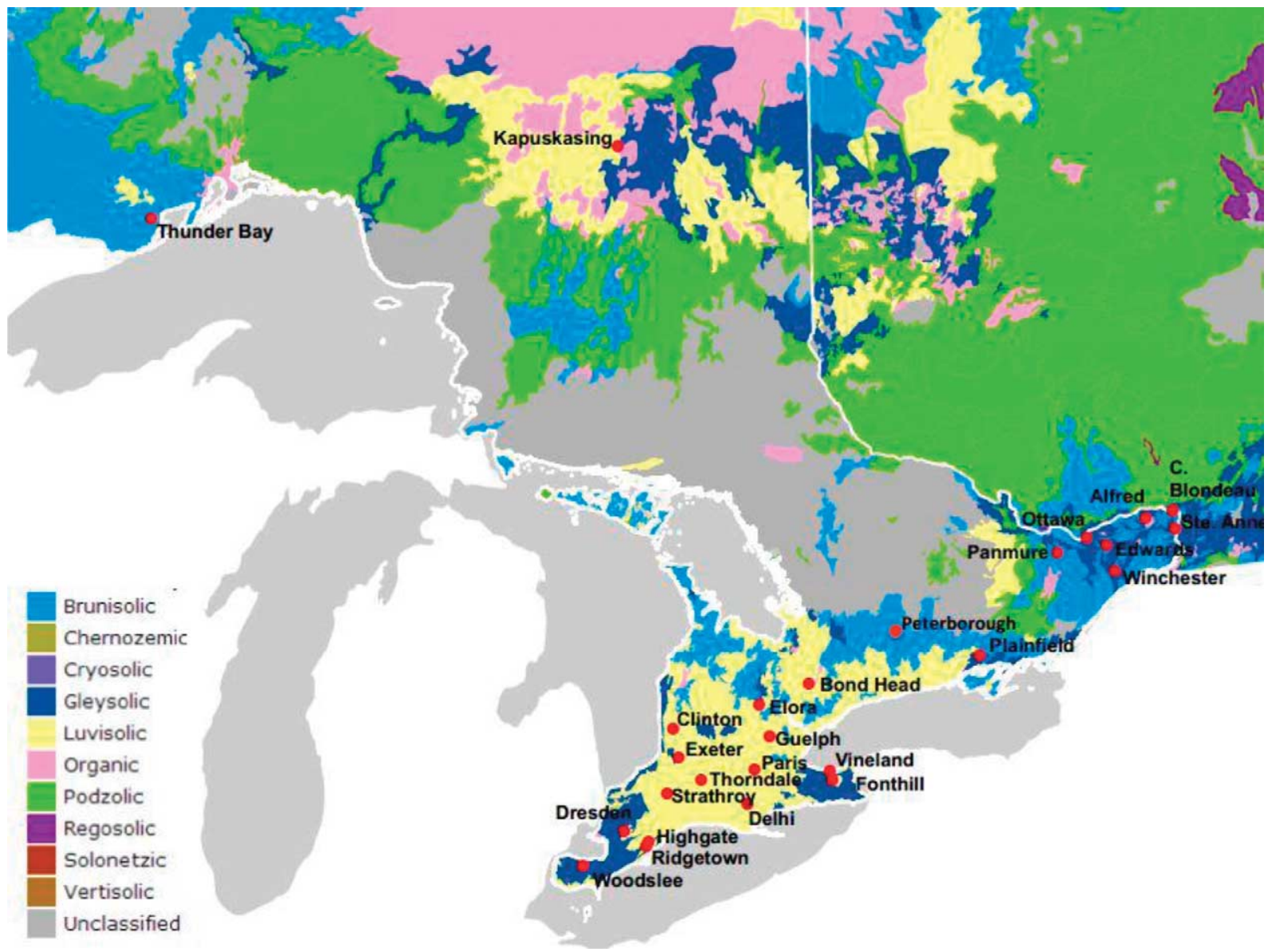

Fig. 1. Soil classification map of southern Ontario with past and present long-term agronomic research sites.

and Post 2002; Luo et al. 2010). The effect of CT vs. NT on SOC in the 0 - to $10-\mathrm{cm}$ and $0-$ to $20-\mathrm{cm}$ depth ranges included only four sites, which included two Gleysols, one Luvisol and one Brunisol, whereas the 0- to $45-\mathrm{cm}$ depth range had a total of eight sites. The four soils that only had data for the 0 - to $45-\mathrm{cm}$ depth range had three soils from the Luvisol group and only one soil from the Gleysolic group (Table 4). Thus, the four sites with 0 - to $10-\mathrm{cm}$ and 0 - to $20-\mathrm{cm}$ data were not representative of the eight sites with 0 - to $45-\mathrm{cm}$ data with regards to soil classification or SOC, and a comparison among depth ranges was limited. Readers should not to compare SOC values among these depths (Table 4). Regardless, the greater sample size within the 0 - to 45 -cm depth $(n=62)$ than shallower depth ranges $(n=23)$ allowed for greater power for SOC estimates within the 0 - to $45-\mathrm{cm}$ depth range.

Overall, most Ontario publications have concluded that NT redistributed SOC within the soil profile but did not increase SOC storage beyond the plough layer (i.e., $>20 \mathrm{~cm}$ ) (Deen and Kataki 2003; Murage et al. 2007a; Angers and Eriksen-Hamel 2008; Yang et al. 2008; Shi et al. 2011). Deen and Kataki (2003) found that NT practices after $25 \mathrm{yr}$ had higher SOC concentrations compared with $\mathrm{CT}$ only for the surface layer at Elora, but not for the $0-$ to $60-\mathrm{cm}$ depth. No-till practice produced SOC stratification at Woodslee, with greater surface SOC but lower SOC in the 10- to $30-\mathrm{cm}$ layer after 16 yr (Shi et al. 2011). At Delhi, similar SOC depth stratification was observed as NT increased SOC stocks in the $0-$ to $5-\mathrm{cm}$ depth, but tillage did not alter SOC in the $0-$ to $20-\mathrm{cm}$ soil depth (Murage et al. 2007a). To detect tillage-induced differences in soil $\mathrm{C}$ beyond the plough layer at sites including Woodslee and Elora after 16 and $23 \mathrm{yr}$, an impractical or expensive amount of minimum core samples may be required (Yang et al. 2008). Other eastern Canadian trials have shown lower SOC in NT than CT at depths below the plough layer (i.e., $>20 \mathrm{~cm}$ depth) (Angers et al. 1997; VandenBygaart et al. 2003). The accumulation of SOC at the plough layer in CT soils in the cool, humid climates of eastern Canada was suggested to be due to restricted crop residue decomposition after soil inversion with tillage (Angers et al. 1997; Angers and Eriksen-Hamel 2008). Thus, tillage is hypothesized to influence SOC dynamics via the change in soil moisture, temperature, structure, and the mixing of plant residues into soil. However, 


\begin{tabular}{|c|c|c|c|c|c|c|c|c|c|}
\hline \multirow[b]{4}{*}{ Site } & \multicolumn{9}{|c|}{ Soil depth $(\mathrm{cm})$} \\
\hline & \multicolumn{3}{|c|}{$0-10(n=23)$} & \multicolumn{3}{|c|}{$0-20(n=23)$} & \multicolumn{3}{|c|}{$0-45(n=62)$} \\
\hline & \multicolumn{9}{|c|}{ Mean change in soil organic $C^{Z}$} \\
\hline & $\begin{array}{c}\mathrm{Net} \\
\left(\mathrm{Mg} \mathrm{C} \mathrm{ha}^{-1}\right)\end{array}$ & Relative (\%) & $\begin{array}{c}\text { Rate } \\
\left(\mathrm{g} \mathrm{C} \mathrm{m}^{-2} \mathrm{yr}^{-1}\right)\end{array}$ & $\begin{array}{c}\mathrm{Net} \\
\left(\mathrm{Mg} \mathrm{C} \mathrm{ha}^{-1}\right)\end{array}$ & Relative (\%) & $\begin{array}{c}\text { Rate } \\
\left(\mathrm{g} \mathrm{C} \mathrm{m}^{-2} \mathrm{yr}^{-1}\right)\end{array}$ & $\begin{array}{c}\mathrm{Net} \\
\left(\mathrm{Mg} \mathrm{C} \mathrm{ha}^{-1}\right)\end{array}$ & Relative (\%) & $\begin{array}{c}\text { Rate } \\
\left(\mathrm{g} \mathrm{C} \mathrm{m}^{-2} \mathrm{yr}^{-1}\right)\end{array}$ \\
\hline Clinton $^{y}$ & $-6.8 a$ & $-14 a b$ & $-34 a b$ & $-11 a$ & $-9.9 b$ & $-55 a b$ & $-13 b$ & $-8.2 b c$ & $-68 b c$ \\
\hline Dresden & & & & & & & $-1.3 a b$ & $-5.2 a b c$ & $-8.8 a b c$ \\
\hline $\begin{array}{l}\text { Elora } \\
\text { Paris }\end{array}$ & $-1.2 a$ & $-4.5 a$ & $-6.0 a$ & $1.8 a$ & $4.7 a$ & $5.1 a$ & 4.6a & $6.5 a b$. & $18 a$ \\
\hline $\begin{array}{l}\text { Paris } \\
\text { Ridgetown }\end{array}$ & $-7.4 a$ & $-22 b$ & $-49 b$ & $-10 a$ & $-16 b$ & $-67 b$ & $\begin{array}{c}0.30 a \\
-16 b\end{array}$ & $\begin{array}{l}-3.0 b c^{x} \\
-15 c\end{array}$ & $\begin{array}{r}2.0 a b \\
-104 c\end{array}$ \\
\hline Strathroy & & & & & & & $7.8 a$ & $16 a$ & $52 a$ \\
\hline Thorndale & & & & & & & $4.9 a$ & $1.8 a b c$ & $32 a$ \\
\hline Woodslee & $-6.9 a$ & $-20 a b$ & $-43 a b$ & $-3.4 a$ & $-5.7 a b$ & $-21 a b$ & $-4.3 a b$ & $-6.9 a b c$ & $-28 a b c$ \\
\hline Mean & -5.5 & -15 & -33 & -6.5 & -8.0 & & -1.2 & -0.83 & -6.9 \\
\hline $95 \%$ Confidence Interval & -8.0 to -3.1 & -19 to -10 & -47 to -19 & -11 to -1.8 & -13 to -2.7 & -66 to -15 & -4.8 to 2.5 & -5.7 to 4.1 & -29 to 15 \\
\hline Standard error & 1.23 & 2.4 & 7.1 & 2.43 & 2.69 & 13.2 & 1.85 & 2.51 & 11.17 \\
\hline
\end{tabular}

${ }^{2}$ Negative values indicate lower soil organic $\mathrm{C}$ with tillage compared with no-till.

${ }^{y}$ Publications include: Gregorich et al. (1996), Wanniarachchi et al. (1999) Yang and Kay (2001b), Gregorich et al. (2001), VandenBygaart et al. (2010), Deen and Kataki (2003), VandenBygaart and Kay (2004), Yang et al. (2008), Shi et al. (2011) and Van Eerd et al. (2014).

${ }^{x}$ An outlier was detected within the Paris data for soil organic C relative change $(\%)$ based on Lund's test and was not discarded (the presence of the outlier explains the incongruence in sign among integers for the $0-45 \mathrm{~cm}$ data at Paris).

$a-c$ In each column, means followed by different letters indicate a significant difference among sites with a LSD test at $P<0.05$. 
interpretations of how tillage affects SOC may differ depending on soil sampling depth range.

For the 0 - to $45-\mathrm{cm}$ profile, the presented Ontario meta-analysis showed CT had lower SOC by $6.9 \mathrm{~g} \mathrm{C} \mathrm{m}^{-2}$ $\mathrm{yr}^{-1}$ compared with NT (Table 4), but the treatment effect (CT) was not considered significant because the mean \pm standard error (11.17) overlapped with zero. The SOC variability was similar between NT and CT. In contrast with our analysis, a compendium by Vanden Bygaart et al. (2003) found that eastern Canadian CT soils (pooled depths from 15 to $50 \mathrm{~cm}$ profiles) had higher SOC by $7 \mathrm{~g} \mathrm{C} \mathrm{m}^{-2} \mathrm{yr}^{-1}$ ( \pm standard error of 27) compared with NT soils. The discrepancy may be due to the larger range in soil sample depths by VandenBygaart et al. (2003) of $0-15$ to $0-50 \mathrm{~cm}$, compared with $0-30$ to $0-45$ $\mathrm{cm}$ in our study. Within the $0-$ to $45-\mathrm{cm}$ depth range, four of the soils examined had lower SOC contents when tilled (range of -8.8 to $-104 \mathrm{~g} \mathrm{C} \mathrm{m}^{-2} \mathrm{yr}^{-1}$ at Clinton, Dresden, Ridgetown, and Woodslee), whereas four other soils showed that SOC was greater when tilled (range of 2.0 to $52 \mathrm{~g} \mathrm{C} \mathrm{m}^{-2} \mathrm{yr}^{-1}$ at Elora, Paris, Strathroy, and Thorndale) (Table 4). Soil texture and possibly drainage class may determine the effects of tillage on SOC. Of the four sites that showed a reduction in 0 - to $45-\mathrm{cm}$ SOC due to tillage, three (Dresden, Ridgetown, and Woodslee) were Gleysols (Fig. 1). The four sites that showed a gain in 0 - to $45-\mathrm{cm}$ SOC due to tillage (Elora, Paris, Strathroy, and Thorndale) were not Gleysols, but were Brunisols or Luvisols (Fig. 1). Potentially, the long-term disturbance of the soil matrix due to CT vs. NT in Gleysolic soils promoted greater loss of deep SOC $(0-45 \mathrm{~cm})$ than in better-drained soils.

Other factors that impact SOC accumulation or loss may have contributed to different rates of SOC change among sites, such as crop biomass, root exudation, and experimental duration. Although the $0-$ to $45-\mathrm{cm}$ range had a greater sample size than shallower depth ranges (0-10 and 0-20 cm), the effect of tillage on SOC in 0- to $45-\mathrm{cm}$ was more variable than when shallower depth ranges were analyzed, as indicated by site variation, standard errors, and confidence intervals (Table 4). Therefore, the variability of the mean change in SOC analyzed via the mass equivalent basis increased at deeper depth ranges $(0-45 \mathrm{~cm})$ relative to shallower depth ranges $(0-10,0-20 \mathrm{~cm})$.

The 0 - to $45-\mathrm{cm}$ depth range used in the meta-analysis included data from $0-30$ to $0-45 \mathrm{~cm}$ but the variability [i.e., standard error of 1.85 for $\mathrm{Mg} \mathrm{SOC} \mathrm{ha}{ }^{-1}$ (Table 4)] did not consistently increase with depth (i.e., the 0 - to $30-\mathrm{cm}, 0-$ to $40-\mathrm{cm}$, and $0-$ to $45-\mathrm{cm}$ depth ranges had standard errors of $0.94,4.7,1.7$ respectively). Thus, the overall variation in the change in SOC due to CT vs. NT in the 0 - to $45-\mathrm{cm}$ depth range was most likely a reflection of the processes of SOC dynamics at deeper profiles. The processes underlying SOC turnover at deeper depths (i.e., $>45 \mathrm{~cm}$ ) are not well known (Schmidt et al. 2011), but our meta-analysis suggests that the stability of SOC at deeper depths was less predictable than surface SOC. Therefore, researchers should use the sites listed in the inventory (Table 2) to pursue a coordinated study on the effects of long-term tillage management on SOC at deep depths $(>45 \mathrm{~cm})$ extending below the tillage layer.

Interestingly, if the Ridgetown site was removed from the current meta-analysis, CT would have had a positive 0 - to $45-\mathrm{cm}$ SOC change of $5 \mathrm{~g} \mathrm{C} \mathrm{m}^{-2} \mathrm{yr}^{-1}$ ( \pm standard error of 11 ) compared with NT, similar to Vanden Bygaart et al. (2003). Consequently if one site can alter the results to such an extent, clearly a more comprehensive examination is needed to better understand the effect of tillage on SOC in Ontario. Others have shown soil physical, chemical and climatic indices explained significant amounts of variation in soil $\mathrm{N}$ across longterm sites in Canada (Dessureault-Rompré et al. 2010), thus trends in the change of SOC over time may be more consistent within soil type or climate categories in Ontario. The current inventory of long-term sites (Table 2) may be a useful resource for researchers to develop sitespecific predictions for SOC change under tillage regimes within Ontario. Previously, a global meta-analysis showed $48 \mathrm{~g} \mathrm{C} \mathrm{m}^{-2} \mathrm{yr}^{-1}$ more $\mathrm{C}$ sequestered with NT than CT (West and Post 2002). But in the 0- to $40-\mathrm{cm}$ profile, a global meta-analysis of 69 field locations by Luo et al. (2010) found no difference in SOC between longterm NT and CT systems. Although the global reviews inform general predictions (i.e., IPCC models), quantifying the effects of management within smaller regions might be more appropriate for regional policies. Therefore, Ontario policy makers might consider using the presented trends, rather than global reviews, for the effect of CT on SOC storage (Table 4) and for developing best management practices.

\section{Crop Rotation}

The meta-analysis showed a positive effect of rotational cropping on SOC compared with continuous corn (Table 5), and agrees with previous meta-analyses, which found that enhancing long-term crop diversity increased SOC accumulation (Drinkwater et al. 1998; West and Post 2002; Eagle and Olander 2012). However, the treatment effect was not considered significant because the means \pm standard error overlapped with zero (Table 5). The effect of rotation on SOC was similar among Elora, Ridgetown, and Woodslee sites at $0-$ to $10-\mathrm{cm}$ and $0-$ to $20-\mathrm{cm}$ soil depths (Table 5). However, within the $0-$ to $45-\mathrm{cm}$ depth there was variation among sites and rotational corn sequestered $22 \%$ or $20 \mathrm{Mg} \mathrm{C}^{-1}$ more SOC than continuous corn at Woodslee compared with Elora or Ridgetown (Table 5). The difference between sites was likely related to the length of the study and the type of rotational crops. The data from Elora and Ridgetown were after 20 and $11 \mathrm{yr}$, respectively, and included winter wheat, soybean, or winter wheat underseeded with red clover, whereas the Woodslee site had alfalfa rotated with corn for $35 \mathrm{yr}$. Gregorich et al. (2001) observed at 
Woodslee that SOC was higher in an alfalfa-corn rotation compared with continuous corn, especially at depths below the plough layer. Meyer-Aurich et al. (2006) also found higher $\mathrm{C}$ sequestration with corn rotated with alfalfa at Elora, but the lowest soil C levels were in the soybean-corn rotation. In general, SOC storage in Ontario was greater with crop rotation than continuous corn; however, SOC decreased when soybeans replaced corn in the rotation (Table 1). The decrease in SOC was likely due to the reduction in quantity of both above- and below-ground residue as well as the lower $\mathrm{C}: \mathrm{N}$ ratio of soybean compared with corn residues.

Other factors associated with rotational cropping compared with continuous corn such as reduced insect and disease pressure can help increase yield and lead to greater residue biomass and soil $\mathrm{C}$ input (Drury and Tan 1995). Additionally, the variation in the characteristics and composition of different residue types may contribute to the accumulation of SOC. For example, alfalfa has a deep tap root, which may provide $\mathrm{C}$ deeper within the profile where oxygen and microbial activity are typically limited, and therefore contribute to accumulation of SOC (Gregorich et al. 2001). Aromatic C levels were higher below the plough layer in alfalfa rotations, suggesting biological resistance of $\mathrm{C}$ degradation (Gregorich et al. 2001). Also, Comeau et al. (2013) found a slower decay rate in pea (Pisum sativum L.) compared with other crops, and attributed the difference to recalcitrance of pea root residue deposits and root hairs penetrating stable microaggregates.

The effect of rotational cropping on SOC was variable across sites at the deeper profiles $(\geq 45 \mathrm{~cm})$ (Table 1). Carbon contribution from roots due to total root biomass and rhizodeposition is not well known for many crops (Johnson et al. 2006; Comeau et al. 2013) and warrants future research. Thus, researchers should use the presented inventory (Table 2) to initiate coordinated studies on how crop rotations, root biomass and distribution influences SOC storage at deeper depths $(\geq 45 \mathrm{~cm})$ in Ontario.

\section{Nitrogen Fertilizer}

Ridgetown and Woodslee were the only sites included in meta-analysis to investigate the effect of $\mathrm{N}$ fertilization on SOC storage in corn rotations (Table 1) because they were the only studies that reported SOC on an equivalent mass basis. The effect of $\mathrm{N}$ fertilizer to the corn crop on SOC appeared significant in the 0 - to $45-\mathrm{cm}$ depth $\left(11 \mathrm{~g} \mathrm{C} \mathrm{m}^{2} \mathrm{yr}^{-1}\right)$ but not at shallower layers $(-2.1$ to $1.5 \mathrm{~g} \mathrm{C} \mathrm{m}^{2} \mathrm{yr}^{-1}$ ) (Table 6). Nitrogen fertilizer likely facilitated increased SOC sequestration due to increased primary production and thereby greater residue $\mathrm{C}$ inputs (Zhang et al. 2012), but research should investigate why the effect on SOC was most pronounced at deeper depths (up to or $>45 \mathrm{~cm}$ ). 
Although the corn $\mathrm{N}$ fertilization experiment was $12 \mathrm{yr}$ longer at Woodslee than Ridgetown, the meta-analysis showed that the sites had similar positive effect of $\mathrm{N}$ fertilization on SOC storage (Table 6). The climatic and soil characteristics were similar between the Ridgetown and Woodslee sites, as the sites are within $100 \mathrm{~km}$ of each other, on poorly drained Brookston clay loam soils, and have corn-based cropping sequences. In a global review, Alvarez (2005) concluded that soil C sequestration increased with $\mathrm{N}$ application. Although Khan et al. (2007) reported a long-term $(51 \mathrm{yr})$ decline in SOC storage in fertilized continuous corn plots, it is critical to recognize that the choice of baseline can alter the results (Lal 2009). Lal (2009) showed that $\mathrm{N}$ fertilizer increased SOC storage based on a treatment comparison between unfertilized and fertilized plots used in the study by Khan et al. (2007). Including other Ontario long-term $\mathrm{N}$ fertilization trials could strengthen the conclusions regarding effect on SOC with $\mathrm{N}$ fertilizer regimes. Fertilizer trials are presently underway at seven other experiments in Ontario including Ottawa, Alfred, Delhi, Elora, Ridgetown, Peterborough, and Thunder Bay (Table 2). Thus, there is future potential for synthesized Ontario research on $\mathrm{N}$ fertilization and SOC.

\section{CONCLUSIONS}

Improving the sustainability of Ontario's agriculture depends in part on knowledge gained by long-term cropping practices on SOC. Ontario has a variety of long-term research plots focused on land management and soil productivity; however, research must be synthesized to understand the effects of management on SOC storage and to develop best practices to prevent soil degradation. Our review of long-term agricultural management effects on SOC in Ontario indicated trends towards higher SOC with NT than CT, crop rotation than continuous corn, and $\mathrm{N}$ fertilizer than none, similar to previous research. However, SOC loss or gain from tillage practices may be determined by soil classification, texture, and drainage in the 0 - to 45-depth range. Management effects on SOC were more variable within deeper profiles $(0-45 \mathrm{~cm})$ than within shallower $(0-10$ and $0-20 \mathrm{~cm}$ ) profiles. Thus, the stability of deep SOC may be less predictable than shallower depths, and researchers are encouraged to use the inventory of nine Ontario sites and 18 active experimental trials (Table 2) to pursue coordinated studies on the effects of long-term management on SOC at deeper depths $(>45 \mathrm{~cm})$.

\section{ACKNOWLEDGEMENTS}

The authors gratefully thank Adam Hayes and Anne Verhallen for making SOC analysis at Ridgetown possible and for valuable comments on the initial draft. For important comments which improved the manuscript, the authors acknowledge the peer-reviewers and editors. 
Alvarez, R. 2005. A review of nitrogen fertilizer and conservation tillage effects on soil organic carbon storage. Soil Use Manage. 21: 38-52.

Angers, D. A. and Eriksen-Hamel, N. S. 2008. Full-inversion tillage and organic carbon distribution in soil profiles: A metaanalysis. Soil Sci. Soc. Am. J. 72: 1370-1374.

Angers, D., Bolinder, M., Carter, M., Gregorich, E., Drury, C., Liang, B., Voroney, R. P., Simard, R., Donald, R. and Beyaert, R. 1997. Impact of tillage practices on organic carbon and nitrogen storage in cool, humid soils of eastern Canada. Soil Tillage Res. 41: 191-201.

Ball-Coelho, B. R. and Roy, R. C. 1997. Overseeding rye into corn reduces $\mathrm{NO}_{3}$ leaching and increases yields. Can. J. Soil Sci. 77: 443-451.

Ball-Coelho, B. R., Roy, R. C. and Bruin, A. J. 2005. Longterm effects of late-summer overseeding on corn grain yield and nitrogen balance. Can. J. Plant Sci. 85: 543-554.

Ball-Coelho, B. R., Roy, R. C. and Swanton, C. J. 1998. Tillage alters corn root distribution in coarse-textured soil. Soil Tillage Res. 45: 237-249.

Beyaert, R. P. and Voroney, R. P. 2011. Estimation of decay constants for crop residues measured over 15 years in conventional and reduced tillage systems in a coarse-textured soil in southern Ontario. Can. J. Soil Sci. 91: 985-995.

Bolinder, M. A., Angers, D. A., Gregorich, E. G. and Carter, M. R. 1999. The response of soil quality indicators to conservation management. Can. J. Soil Sci. 79: 37-45.

Bolinder, M. A., VandenBygaart, A. J., Gregorich, E. G., Angers, D. A. and Janzen, H. H. 2006. Modelling soil organic carbon stock change for estimating whole-farm greenhouse gas emissions. Can. J. Soil Sci. 86: 419-429.

Bolton, E. F., Aylesworth, J. W. and Hore, F. R. 1970. Nutrient losses through tile drains under three cropping systems and two fertilizer levels on a Brookston clay soil. Can. J. Soil Sci. 50: 275-279.

Campbell, C. A., Juma, N. G., Zentner, R. P., Smith, E., Izaurralde, R. C., Robertson, J. A., Dyck, M. and Janzen, H. H. 2012. A bibliography of scientific publications based on long-term crop rotation studies in the Canadian prairies. Prairie Soils Crops 5: 7-29. [Online] Available: http://www. prairiesoilsandcrops.ca [2014 May. 13].

Cardinael, R., Thevathasan, N., Gordon, A., Clinch, R., Mohammed, I. and Sidders, D. 2012. Growing woody biomass for bioenergy in a tree-based intercropping system in southern Ontario, Canada. Agroforest. Syst. 86: 279-286.

Carter, M. R. 2005. Long-term effects on cool-season soybean in rotation with barley, soil proerties, and carbon and nitrogen storage for fine sandy loams in the humid climate of Atlantic Canada. Soil Tillage Res. 81: 109-120.

Carter, M. R., Gregorich, E. G., Angers, D. A., Donald, R. and Bolinder, M. A. 1998. Organic C and N storage, and organic C fractions, in adjacent cultivated and forested soils of Eastern Canada. Soil Tillage Res. 47: 253-261.

Cartier, L. C. 2011. Nitrogen dynamics as affected by cover crop type, planting date and rye biomass spring removal in a cover crop - cucumber rotation. M.Sc. thesis 2011. University of Guelph, Guelph, ON.

Clinch, R. L., Thevathasan, N. V., Gordon, A. M., Volk, T. A. and Sidders, D. 2009. Biophysical interactions in a short rotation willow intercropping system in southern Ontario, Canada. Agric. Ecosyst. Environ. 131: 61-69.

Comeau, L. P., Lemke, R. L., Knight, J. D. and BedardHaughn, A. 2013. Carbon input from ${ }^{13} \mathrm{C}$-labeled crops in four soil organic matter fractions. Biol. Fertil. Soils. [Online] Available: http://link.springer.com/article/10.1007/s00374-0130816-4\#page-1 [2013 Aug. 28].

Coote, D. and Ramsey, J. 1983. Quantification of the effects of over 35 years of intensive cultivation on four soils. Can. J. Soil Sci. 63: 1-14.

Crolla, A., Kinsley, C. and Pattey, E. 2013. Land application of digestate. Ch. 13 in A. Wellinger, J. Murphy, and D. Baxter, eds. The biogas handbook: Science, production and applications. Woodhead Publishing, Cambridge, UK. 512 pp.

Debreczeni, K. and Körschens, M. 2003. Long-term field experiments of the world. Arch. Acker-Pflanzenbau Bodenkd. 49: 465-483.

Deen, W. and Kataki, P. 2003. Carbon sequestration in a longterm conventional versus conservation tillage experiment. Soil Tillage Res. 74: 143-150.

Dessureault-Rompré, J., Zebarth, B. J., Burton, D. L., Sharifi, M., Cooper, J., Grant, C. A. and Drury, C. F. 2010. Relationships among mineralizable soil nitrogen, soil properties, and climatic indices. Soil Sci. Soc. Am. J. 74: 1218-1227.

Deubel, A., Hofmann, B. and Orzessek, D. 2011. Long-term effects of tillage on stratification and plant availability of phosphate and potassium in a loess chernozem. Soil Tillage Res. 117: 85-92.

Drinkwater, L. E., Wagoner, P. and Sarrantonio, M. 1998. Legume-based cropping systems have reduced carbon and nitrogen losses. Nature 396: 262-265.

Drury, C. F. and Tan, C. S. 1995. Long-term (35 years) effects of fertilization, rotation and weather on corn yields. Can. J. Plant Sci. 75: 355-362.

Drury, C. F., Reynolds, W. D., Yang, X. M., Welacky, T. W., McLaughlin, N. B., Calder, W. and Grant, C. A. 2012. Nitrogen source, application time and tillage effects on soil $\mathrm{N}_{2} \mathrm{O}$ emissions and corn grain yields. Soil Sci. Soc. Am. J. 76: 1268-1279.

Drury, C. F., Yang, X. M., Reynolds, W. D. and Tan, C. S. 2004. Influence of crop rotation and aggregate size on carbon dioxide production and denitrification. Soil Tillage Res. 79: 87-100.

Eagle, A. J. and Olander, L. P. 2012. Greenhouse Gas mitigation with agricultural land management activities in the United States - A side-by-side comparison of biophysical potential. Adv. Agron. 115: 79-179.

Ellert, B. and Gregorich, E. G. 1996. Storage of carbon, nitrogen and phosphorus in cultivated and adjacent forested soils of Ontario. Soil Sci. 161: 587-603.

Franchini, J. C., Crispino, C. C., Souza, R. A., Torres, E. and Hungria, M. 2007. Microbiological parameters as indicators of soil quality under various soil management and crop rotation systems in southern Brazil. Soil Tillage Res. 92: 18-29.

Fuentes, M., Govaerts, B., De León, F., Hidalgo, C., Dendooven, L., Sayre, K. D. and Etchevers, J. 2009. Fourteen years of applying zero and conventional tillage, crop rotation and residue management systems and its effect on physical and chemical soil quality. Eur. J. Agron. 30: 228-237.

Gillespie, A. W., Diochon, A., Ma, B. L., Morrison, M. J., Kellman, L., Walley, F. L., Regier, T. Z., Chevrier, D., Dynes, J. J. and Gregorich, E. G. 2014. Nitrogen input quality changes the biochemical composition of soil organic matter stabilized in the fine fraction: A long-term study. Biogeochemistry 117: 337-350. 
Gregorich, E. G., Drury, C. and Baldock, J. A. 2001. Changes in soil carbon under long-term maize in monoculture and legume-based rotation. Can. J. Soil Sci. 81: 21-31.

Gregorich, E. G., Liang, B. C., Ellert, B. H. and Drury, C. F. 1996. Fertilization effects on soil organic matter turnover and corn residue C storage. Soil Sci. Soc. Am. J. 60: 472-476.

Gregorich, E. G., Carter, M. R., Angers, D. A. and Drury, C. F. 2009. Using a sequential density and particle-size fractionation to evaluate $\mathrm{C}$ storage in the profile of tilled and no-till soils in eastern Canada. Can. J. Soil Sci. 89: 255-267.

Gregorich, E. G., Rochette, P., Hopkins, D. W., McKim, U. F. and St-Georges, P. 2006. Tillage induced environmental conditions in soil and substrate limitation determine biogenic gas production. Soil Biol. Biochem. 38: 2614-2628.

Halpern, M. T., Whalen, J. K. and Madramootoo, C. A. 2010. Long-term tillage and residue management influences soil carbon and nitrogen dynamics. Soil Sci. Soc. Am. J. 74: 1211-1217.

Intergovernmental Panel on Climate Change. 1997. Revised 1996 IPCC guidelines for national greenhouse gas inventories: reference manual. IPCC, Geneva, Swithzerland.

Janzen, H. H. 1995. The role of long-term sites in agroecological research: A case stud. Can. J. Soil Sci. 75: 123-133.

Johnson, J. M. F., Allmaras, R. R. and Reicosky, D. C. 2006. Estimating source carbon from crop residues, roots and rhizodeposits using the national grain-yield database. Agron. J. 98: 622-636.

Khan, S., Mulvaney, R., Ellsworth, T. and Boast, C. 2007. The myth of nitrogen fertilization for soil carbon sequestration. J. Environ. Qual. 36: 1821-1832.

Lafond, G. P. and Harker, K. N. 2012. Long-term cropping studies on the Canadian Prairies: An introduction. Prairie Soils Crops 5: 1-6.

Lal, R. 2009. Challenges and opportunities in soil organic matter research. Eur. J. Soil Sci. 60: 158-169.

Lipsey, M. W. and Wilson, D. B. 2000. Practical meta-analysis. Sage Publications, Thousand Oaks, CA. 254 pp.

Luo, Z., Wang, E. and Sun, O. J. 2010. Can no-tillage stimulate carbon sequentration in agricultural soils? A metaanalysis of paired experiments. Agric. Ecosyst. Environ. 139: 224-231.

Ma, B. L., Dwyer, L. M. and Gregorich, E. G. 1999a. Soil nitrogen amendment effects on nitrogen uptake and grain yield of maize. Agron. J. 91: 650-656.

Ma, B. L., Dwyer, L. M. and Gregorich, E. G. 1999b. Soil nitrogen amendment effects on seasonal nitrogen mineralization and nitrogen cycling in maize production. Agron. J. 91: 1003-1009.

Ma, B. L, Liang, B., Biswas, D. K., Morrison, M. J. and McLaughlin, N. B. 2012. The carbon footprint of maize production as affected by nitrogen fertilizer and maize-legume rotations. Nutr. Cycling Agroecosyst. 94: 15-31.

Ma, B. L., Subedi, K. D. and Liu, A. 2006. Variations in grain nitrogen removal associated with management practices in maize production. Nutr. Cycling Agroecosyst. 76: 1385-1314. McGonigle, T. P., Miller, M. M. and Young, D. 1999. Mycorrhizae, crop growth, and crop phosphorus nutrition in maize-soybean rotations given various tillage treatments. Plant Soil 210: 33-42.

Meyer-Aurich, A., Weersink, A., Janovicek, K. and Deen, W. 2006. Cost efficient rotation and tillage options to sequester carbon and mitigate $\mathrm{GHG}$ emissions from agriculture in Eastern Canada. Agric. Ecosyst. Environ. 117: 119-127.
Mueller, L., Kay, B. D., Deen, B., Hu, C., Zhang, Y., Wolff, M., Eulenstein, F. and Schindler, U. 2009b. Visual assessment of soil structure: Part II. Implications of tillage, rotation and traffic on sites in Canada, China and Germany. Soil Tillage Res. 103: 188-196.

Mueller, L., Kay, B. D., Hu, C., Li, Y., Schindler, U., Behrendt, A., Shepherd, T. G. and Ball, B. C. 2009a. Visual assessment of soil structure: Evaluation of methodologies on sites in Canada, China and Germany: Part I: Comparing visual methods and linking them with soil physical data and grain yield of cereals. Soil Tillage Res 103: 178-187.

Murage, E. W. and Voroney, R. P. 2008. Distribution of organic carbon in the stable soil humic fractions as affected by tillage management. Can. J. Soil Sci. 88: 99-106.

Murage, E. W., Voroney, R. P., Kay, B. D., Deen, B. and Beyaert, R. P. 2007a. Dynamics and turnover of soil organic matter and its density fractions as affected by tillage. Soil Sci. Soc. Am. J. 71: 1363-1370.

Murage, E. W., Voroney, R. P. and Beyaert, R. P. 2007b. Turnover of carbon in the free light fraction with and without charcoal as determined using the $13 \mathrm{C}$ natural abundance method. Geoderma 138: 133-143.

Munkholm, L. J., Heck, R. J. and Deen, B. 2013. Long-term rotation and tillage effects on soil structure and crop yield. Soil Tillage Res. 127: 85-91.

Ontario. 2009. Environmental Commission: Building resilience. Comissioner: The Honourable Gord Miller, Toronto, ON. [Online] Available: http://www.eco.on.ca/eng/uploads/ eng_pdfs/2009/ar2008.pdf.

O'Reilly, K. A., Robinson, D. E., Vyn, R. J. and Van Eerd, L. L. 2011. Weed populations, sweet corn yield and economics following fall cover crops. Weed Technol. 25: 374-384.

O'Reilly, K. A., Lauzon, J. D., Vyn, R. J. and Van Eerd, L. L. 2012. Nitrogen cycling, profit margins and sweet corn yield under fall cover crop systems. Can. J. Soil Sci. 92: 353-365.

Peichl, M., Thevathasan, N. V., Gordon, A. M., Huss, J. and Abohassan, R. A. 2006. Carbon sequestration potentials in temperate tree-based intercropping systems, southern Ontario, Canada. Agrofor. Syst. 66: 243-257.

Powlson, D. S., Smith, P., Coleman, K., Smith, J. U., Glendining, M. J., Körschens, M. and Franko, U. 1998. A European network of long-term sites for studies on soil organic matter. Soil Tillage Res. 47: 263-274.

Reeleder, R. D., Miller, J. J., Ball-Coelho, B. R. and Roy, R. C. 2006. Impacts of tillage, cover crop, and nitrogen on populations of earthworms, microarthropods, and soil fungi in a cultivated fragile soil. Appl. Soil Ecol. 33: 243-257.

Schmidt, M. W., Torn, M. S., Abiven, S., Dittmar, T., Guggenberger, G., Janssens, I. A. and Trumbore, S. E. 2011. Persistence of soil organic matter as an ecosystem property. Nature 478: 49-56.

Sharifi, M., Zebarth, B. L., Burton, D. L., Grant, C. A., Bittman, S., Drury, C. F., McConkey, B. G. and Ziadi, N. 2008. Response of soil potentially mineralizable $\mathrm{N}$ and indices of $\mathrm{N}$ availability to tillage system. Soil Sci. Soc. Am. J. 72: 1124-1131.

Shi, X., Yang, X., Drury, C. F., Reynolds, W. D., McLaughlin, N. B., Welacky, T. W. and Zhang, X. 2011. Zone tillage impacts on organic carbon of a clay loam in southwestern Ontario. Soil Sci. Soc. Am. J. 75: 1083-1089.

Shi, X. H., Yang, X. M., Drury, C. F., Reynolds, W. D., McLaughlin, N. B. and Zhang, X. 2012. Impact of ridge tillage 
on organic carbon and selected physical properties of a clay loam in Southwestern Ontario. Soil Tillage Res. 120: 1-7.

Smith, P. 2004. How long before a change in soil organic carbon can be detected? Glob. Change Biol. 10: 1878-1883.

Swanton, C. J., Shrestha, A., Roy, R. C., Ball Coelho, B. and Knezevic, S. Z. 1999. Effect of tillage systems, nitrogen, and cover crop on the composition of weed flora in Zea mays. Weed Sci. 47: 454-461.

Thevathasan, N. V. and Gordon, A. M. 2004. Ecology of tree intercropping systems in the North temperate region: Experiences from southern Ontario, Canada. Agrofor. Syst. 61: 257-268.

Topp, G. C., Dow, B., Edwards, M., Gregorich, E. G., Curnoe, W. E. and Cook, F. J. 2000. Oxygen measurements in the root zone facilitated by TDR. Can. J. Soil Sci. 80: 33-41.

VandenBygaart, A. and Angers, D. 2006. Towards accurate measurements of soil organic carbon stock change in agroecosystems. Can. J. Soil Sci. 86: 465-471.

VandenBygaart, A. and Kay, B. 2004. Persistence of soil organic carbon after plowing a long-term no-till field in southern Ontario, Canada. Soil Sci. Soc. Am. J. 68: 1394-1402. VandenBygaart, A., Bremer, E., McConkey, B., Ellert, B., Janzen, H., Angers, D., Carter, M., Drury, C., Lafond, G. and McKenzie, R. 2011. Impact of sampling depth on differences in soil carbon stocks in long-term agroecosystem experiments. Soil Sci. Soc. Am. J. 75: 226-234.

VandenBygaart, A., Bremer, E., McConkey, B., Janzen, H., Angers, D., Carter, M., Drury, C., Lafond, G. and McKenzie, R. 2010. Soil organic carbon stocks on long-term agroecosystem experiments in Canada. Can. J. Soil Sci. 90: 543-550.

VandenBygaart, A., Gregorich, E. and Angers, D. 2003. Influence of agricultural management on soil organic carbon: A compendium and assessment of Canadian studies. Can. J. Soil Sci. 83: 363-380.

VandenBygaart, A., Yang, X., Kay, B. and Aspinall, J. 2002. Variability in carbon sequestration potential in no-till soil landscapes of southern Ontario. Soil Tillage Res. 65: 231-241. Van Eerd, L. L., Congreves, K. A., Hayes, A., Verhallen, A. and Hooker, D. C. 2014. Long-term tillage and crop rotation effects on soil quality, organic carbon, and total nitrogen. Can. J. Soil Sci. 94: 303-315.

Vyn, T. J. and Raimbault, B. A. 1993. Long-term effect of five tillage systems on corn response and soil structure. Agron. J. 85: 1074-1079.
Walbridge, M. R. and Shafer, S. S. 2011. A long-term agroecosystem research (LTAR) network for agriculture. The Fourth Interagency Conference on Research in the Watersheds, 2011 Sep. 26-30, Fairbanks, AK.

Wanniarachchi, S., Voroney, R. P., Vyn, T. J., Beyaert, R. P. and MacKenzie, A. 1999. Tillage effects on the dynamics of total and corn-residue-derived soil organic matter in two southern Ontario soils. Can. J. Soil Sci. 79: 473-480.

West, T. O. and Post, W. M. 2002. Soil organic carbon sequestration rates by tillage and crop rotation: A global data analysis. Soil Sci. Soc. Am. J. 66: 1930-1946.

Wilson, D. B. 2005. Meta-analysis macros for SAS, SPSS, and Stata. [Online] Available: http://mason.gmu.edu/ dwilsonb/ ma.html [2013 Dec.].

Xie, H. T., Yang, X. M., Drury, C. F., Yang, J. Y. and Zhang, X. D. 2011. Predicting soil organic carbon and total nitrogen using mid- and near-infrared spectra for Brookston clay loam soil in Southwestern Ontario, Canada. Can. J. Soil Sci. 91: 53-63.

Yang, X. and Kay, B. 2001a. Rotation and tillage effects on soil organic carbon sequestration in a typic Hapludalf in Southern Ontario. Soil Tillage Res. 59: 107-114.

Yang, X. and Kay, B. 2001b. Impacts of tillage practices on total, loose-and occluded-particulate, and humified organic carbon fractions in soils within a field in southern Ontario. Can. J. Soil Sci. 81: 149-156.

Yang, X. M., Drury, C. F., Wander, M. M. and Kay, B. D. 2008. Evaluating the effect of tillage on carbon sequestration using the minimum detectable difference concept. Pedosphere 18: $421-430$.

Zhang, B., Yang, X. M., Drury, C. F., Reynolds, W. D., He, H. and Zhang, X. 2012. Effects of 49 years of fertilization on the distribution and accumulation of soil carbon under corn cultivation. Can. J. Soil Sci. 92: 835-839.

Zhang, J. X., Xue, A. G., Cober, E. R., Morrison, M. J., Zhang, H. J., Zhang, S. Z. and Gregorich, E. 2013. Prevalence, pathogenicity and cultivar resistance of Fusarium and Rhizoctonia species causing soybean root rot. Can. J. Plant Sci. 93: $221-236$.

Zhang, Z. D., Yang, X. M., Drury, C. F., Reynolds, W. D. and Zhao, L. P. 2010. Mineralization of active soil organic carbon in particle size fractions of a Brookston clay soil under notillage and mouldboard plough tillage. Can. J. Soil Sci. 90: 551-557. 